Article

\title{
Nekhoroshev Stability for the Dirichlet Toda Lattice
}

\author{
Andreas Henrici \\ Institut für Angewandte Mathematik und Physik, ZHAW School of Engineering, Technikumstrasse 9, Postfach, \\ CH-8400 Winterthur, Switzerland; andreas.henrici@zhaw.ch; Tel.: +41-589-346-292
}

Received: 3 September 2018; Accepted: 11 October 2018; Published: 16 October 2018

\begin{abstract}
In this work, we prove a Nekhoroshev-type stability theorem for the Toda lattice with Dirichlet boundary conditions, i.e., with fixed ends. The Toda lattice is a member of the family of Fermi-Pasta-Ulam (FPU) chains, and in view of the unexpected recurrence phenomena numerically observed in these chains, it has been a long-standing research aim to apply the theory of perturbed integrable systems to these chains, in particular to the Toda lattice which has been shown to be a completely integrable system. The Dirichlet Toda lattice can be treated mathematically by using symmetries of the periodic Toda lattice. Precisely, by treating the phase space of the former system as an invariant subset of the latter one, namely as the fixed point set of an important symmetry of the periodic lattice, the results already obtained for the periodic lattice can be used to obtain analogous results for the Dirichlet lattice. In this way, we transfer our stability results for the periodic lattice to the Dirichlet lattice. The Nekhoroshev theorem is a perturbation theory result which does not have the probabilistic character of related theorems, and the lattice with fixed ends is more important for applications than the periodic one.
\end{abstract}

Keywords: perturbation theory; integrable systems; Fermi-Pasta-Ulam chain

\section{Introduction}

In this paper, we consider perturbations of the Toda lattice with two different kinds of boundary conditions, namely periodic and Dirichlet (fixed ends) boundary conditions. The periodic case is the structurally fundamental one, whereas the Dirichlet case is more imporant for applications; in particular, the famous numerical experiments of Fermi, Pasta, and Ulam with the family of "FPU chains" (see [1]), of which the Toda lattice is a special case, were performed with Dirichlet boundary conditions.

Among the entire family of FPU chains, the Toda lattice has especially strong integrability properties, which makes it possible to obtain results not only in a neighborhood of the equilibrium point, but on the entire phase. This makes it possible to rigorously prove certain stability properties also far away from the equilibrium, i.e., for high energies of the system.

The Toda lattice however should not only be considered as a member of the family of FPU chains; on the contrary, it has been investigated mostly independently from the FPU framework, since it is, as a recent review article [2] states, "a wonderful case study in mechanics and symplectic geometry" with ramifications ranging from representation theory [3] to numerical analysis [4]. Moreover, the Toda lattice is a model with has numerous applications in the physical sciences, ranging from solid state physics [5] and quantum field theory [6] to DNA transcription [7], to mention just a few. Some review articles on the history of the research on the Toda lattice have been published very recently [8-10].

On the other hand, the research on general FPU chains also has its own rich history since the numerical experiments of Fermi, Pasta, and Ulam; since the present paper does not discuss general FPU chains, we refer to the literature for further references, e.g., the overview article [11].

The goal of this paper is the proof of a Nekhoroshev-type result for the Dirichlet Toda lattice. Even though we have already obtained a result of this type for the periodic lattice, we consider the result 
for the lattice with Dirichlet boundary conditions to be of additional interest, since, as just mentioned, most applications of this type of chains have Dirichlet boundary conditions. Besides these two types of boundary conditions, there also exists a rich literature on the infinite Toda lattice, i.e., the lattice with an infinite number of particles without periodicity. For results on the long-time asymptotics of this kind of Toda lattices, using in particular tools from inverse scattering transform and Riemann-Hilbert theory see e.g., [12] and the references therein. The inverse scattering transform as a tool to investigate the dynamics of nonlinear systems was originally developed for the Korteveg-de Vries (KdV) equation [13].

Finally, we consider this result to be an interesting case study for the Nekhoroshev theorem, a major perturbation theory result for integrable systems. It is well-known that the Toda lattice with both types of boundary conditions is an integrable system (for the periodic case see [14-16], for the Dirichlet case see [15]). The Toda lattice is one of a small number of physical examples outside of the realm of celestial mechanics, where the prerequisites of the Nekhoroshev theorem have actually been checked. A better-known stability result for integrable systems is the KAM theorem (see e.g., [17]), whose applicability to this system we have already shown for both types of boundary conditions [18,19]; however, the Nekhoroshev theorem has the advantage of being free from probabilistic elements-recall that the stability statements of the KAM theorem only hold for a majority of initial conditions.

We accomplish our task by embedding the Dirichlet lattice into a periodic lattice of a higher dimension, and the idea is to use the results on the periodic lattice already obtained. However, we cannot directly apply this previous work to the Dirichlet case, since the image of the phase space of the Dirichlet lattice under this embedding is contained in a subset of the periodic phase space not covered by the previous result. Therefore, we have to adapt some of the previous results to the new circumstances. For most of the auxiliary facts needed for our final result, they can be obtained in a similar way as before, and we do not reformulate everything explicitly in the present paper and sometimes refer to the work already done.

\section{Results}

To formally state our results, we first need to introduce the mathematical model of the Toda lattice with various types of boundary conditions. In the periodic case, the lattice with $N$ particles $(N \geq 2)$ is given by the Hamiltonian

$$
H_{\text {Toda }}=\frac{1}{2} \sum_{n=1}^{N} p_{n}^{2}+\alpha^{2} \sum_{n=1}^{N} e^{q_{n}-q_{n+1}}
$$

where $\alpha$ is a positive parameter, $\alpha>0$, and the boundary conditions

$$
\left(q_{n+N}, p_{n+N}\right)=\left(q_{n}, p_{n}\right) \quad \forall n \in \mathbb{Z} .
$$

In the Dirichlet case, the lattice with $N^{\prime}$ particles $\left(N^{\prime} \geq 2\right)$ is given by the Hamiltonian

$$
H_{\text {Toda }}^{(D)}=\frac{1}{2} \sum_{n=1}^{N^{\prime}} p_{n}^{2}+\gamma^{2} \sum_{n=0}^{N^{\prime}} e^{q_{n}-q_{n+1}},
$$

where $\gamma$ again is a positive parameter, $\gamma>0$, and the boundary conditions

$$
q_{0}=q_{N^{\prime}+1}=p_{0}=p_{N^{\prime}+1} \equiv 0 .
$$

We will show in Section 3 how the aforementioned embedding of the model (3) into the model (1) can be accomplished explicitly (for a suitable $N$ depending on the chosen $N^{\prime}$ ).

The main result of this paper is the following:

Theorem 1. There exists an open and dense subset of the phase space of the Toda lattice with Dirichlet boundary conditions on which Nekhoroshev's theorem applies to sufficiently small perturbations of the Dirichlet Toda Hamiltonian. 
We describe the general set-up and give the precise formulation of Nekhoroshev's theorem in Appendix A, thereby closely following [20]; see also the references given in [21]. Nekhoroshev's original work can be found in $[22,23]$. Its main prerequisites are the construction of action variables on the phase space and the convexity of the Hamiltonian with respect to these action variables. We accomplish the tasks of constructing action variables and proving the convexity of the Hamiltonian in Theorems 2 and 3, respectively. We proved the following Theorem in [18]:

Theorem 2. For any fixed $\gamma \in \mathbb{R}$ and $N^{\prime} \geq 2$, the Dirichlet Toda lattice admits a Birkhoff normal form. More precisely, there are (globally defined) canonical coordinates $\left(x_{k}, y_{k}\right)_{1 \leq k \leq N^{\prime}}$ so that $H_{\text {Toda }}^{(D)}$, when expressed in these coordinates, is a function of the action variables $I_{k}=\left(x_{k}^{2}+y_{k}^{2}\right) / 2\left(1 \leq k \leq N^{\prime}\right)$ alone, $H_{\text {Toda }}^{(D)}=\mathcal{H}_{\gamma}(I)$.

Technically, the main result of this paper states that the Hamiltonian $\mathcal{H}_{\gamma}$ is a convex function of the actions variables $\left(I_{k}\right)_{1 \leq k \leq N^{\prime}}$.

Theorem 3. In the open quadrant $\mathbb{R}_{>0}^{N^{\prime}}$, the Hamiltonian $\mathcal{H}_{\gamma}(I)$ introduced in Theorem 2 is a strictly convex function of the action variables $\left(I_{k}\right)_{1 \leq k \leq N^{\prime}}$. Precisely, for any compact subset $U \subseteq \mathbb{R}_{>0}^{N^{\prime}}$ and any compact interval $\left[\alpha_{1}, \alpha_{2}\right] \subseteq \mathbb{R}_{>0}$, there exists $m>0$, such that

$$
\left\langle\partial_{I}^{2} \mathcal{H}_{\gamma}(I) \xi, \xi\right\rangle \geq m\|\xi\|^{2}, \quad \forall \xi \in \mathbb{R}^{N^{\prime}}
$$

for any $I \in U$, and any $\alpha_{1} \leq \gamma \leq \alpha_{2}$.

The convextiy statement (5) shows that the prerequisites of Nekhoroshev's general theorem (see Theorem A1 in Appendix A) are satisfied in the present case. Therefore Theorem 3 implies that Nekhoroshev's Theorem holds for the Dirichlet Toda lattice on $\mathcal{P} \bullet:=\left\{(q, p) \in \mathbb{R}^{2 N^{\prime}} \mid I_{n}(q, p)>0 \forall 1 \leq n \leq N^{\prime}\right\}$, an open and dense subset of $\mathbb{R}^{2 N^{\prime}}$ by Theorem 2 . This then proves our main result, Theorem 1 .

To prove Theorem 3, we use the Birkhoff normal form of the Dirichlet Hamiltonian $\mathcal{H}_{\gamma}$ near $I=0$, also established in [18].

Theorem 4. Near $I=0$, the function $\mathcal{H}_{\gamma}(I)$ introduced in Theorem 2 admits an expansion of the form

$$
\left(N^{\prime}+1\right) \gamma^{2}+\sqrt{2} \gamma \sum_{k=1}^{N^{\prime}} s_{k} I_{k}+\frac{1}{16\left(N^{\prime}+1\right)} \sum_{k=1}^{N^{\prime}} I_{k}^{2}+O\left(I^{3}\right),
$$

with $s_{k}=\sin \frac{k \pi}{2\left(N^{\prime}+1\right)}$. In particular, the Hessian of $\mathcal{H}_{\gamma}(I)$ at $I=0$ is given by

$$
\left.d_{I}^{2} \mathcal{H}_{\gamma}(I)\right|_{I=0}=\frac{1}{32\left(N^{\prime}+1\right)} I d_{N^{\prime}}
$$

As an immediate consequence of (7), we obtain

Corollary 1. Near $I=0, \mathcal{H}_{\gamma}(I)$ ist strictly convex for any $\gamma>0$.

Outside of $I=0$, we argue differently. As mentioned above, we embed the phase space of the Dirichlet lattice into the phase space of the periodic lattice and use an analogous convexity result for the periodic lattice via pullback to the Dirichlet lattice. The image of this embedding is the fixed point set of a certain symmetry map $S$ of the periodic lattice. This fixed point set is a submanifold of the entire phase space of the periodic lattice which is invariant under the evolution induced by the Hamiltonian (1); in this way, this embedding allows us to obtain results on the Dirichlet lattice by exploiting the properties of the symmetry map $S$. 
Related work: As already mentioned, similar results have been obtained for the periodic lattice, see [21], and for other type of perturbation theories, namely the KAM theorem instead of the Nekhoroshev theorem, see [18]. The technique of expressing symmetry properties of a system in terms of action-angle variables and Birkhoff coordinates has developed for the defocusing nonlinear Schrödinger equation, see [24]. The technique of embedding the phase space of a lattice with Dirichlet boundary conditions into the phase space of the corresponding lattice with periodic boundary conditions has been used in the case of arbitrary FPU chains, see $[25,26]$. On perturbations of the Toda lattice in general, there have been published many papers, see e.g., [27-29], usually however applying other techniques than the theorems of KAM and Nekhoroshev which are the main theoretical tool of our approach. The entire work was originally motivated by the pioneering work on a KAM-type result for the KdV equation [30].

Outline of the paper: In Section 3 we describe suitable coordinates for both types of lattices and then the aforementioned embedding of the Dirichlet lattice into the periodic lattice of a higher dimension. In Section 4, we describe some spectral quantities associated with the Toda lattice and their behavior under the symmetry map needed for the embedding described before. This symmetry map also induces a special Riemann surface needed in the sequel, see Section 5; this allows then us to prove Theorem 3. In the appendices, certain lengthy calculations are presented, in addition to some proofs which are very similar to analogous proofs in our previous work [21].

\section{Coordinates and Symmetries}

Here we first describe suitable coordinates for the Dirichlet lattice and then an embedding of the Dirichlet lattice wih $N^{\prime}$ particles into the periodic lattice with $N=2 N^{\prime}+2$ particles.

Coordinates for the Dirichlet lattice As a first step in the Dirichlet case, following Flaschka [14], we introduce the (noncanonical) coordinates

$$
d_{n}:=-p_{n} \in \mathbb{R} \quad\left(0 \leq n \leq N^{\prime}\right), \quad c_{n}:=\gamma e^{\frac{1}{2}\left(q_{n}-q_{n+1}\right)} \in \mathbb{R}_{>0} \quad\left(0 \leq n \leq N^{\prime}\right) .
$$

The boundary conditions (4) imply that $d_{0}=0$ and $\prod_{n=0}^{N^{\prime}} c_{n}=\gamma^{N^{\prime}+1}$; we will identify related Casimir functions below.

In these coordinates the Hamiltonian $H_{T o d a^{\prime}}^{(D)}$ given by (3), takes the simple form

$$
H^{(D)}=\frac{1}{2} \sum_{n=1}^{N^{\prime}} d_{n}^{2}+\sum_{n=0}^{N^{\prime}} c_{n}^{2}
$$

and the equations of motion are, in terms of the $c_{j}$ 's and $d_{j}$ 's defined by (8),

$$
\left\{\begin{array}{l}
\dot{d}_{0}=0 \\
\dot{d}_{n}=c_{n}^{2}-c_{n-1}^{2} \quad\left(1 \leq n \leq N^{\prime}\right), \\
\dot{c}_{0}=\frac{1}{2} c_{0} d_{1} \\
\dot{c}_{n}=\frac{1}{2} c_{n}\left(d_{n+1}-d_{n}\right) \quad\left(1 \leq n \leq N^{\prime}-1\right), \\
\dot{c}_{N^{\prime}}=-\frac{1}{2} c_{N^{\prime}} d_{N^{\prime}} .
\end{array}\right.
$$

We study the system of Equation (10) on the 2(N $\left(N^{\prime}+1\right)$-dimensional phase space

$$
\mathcal{M}^{(D)}:=\mathbb{R}^{N^{\prime}+1} \times \mathbb{R}_{>0}^{N^{\prime}+1} .
$$

This system is Hamiltonian with respect to a suitable nonstandard and degenerate Poisson structure $J^{(D)} \equiv J_{d, c}^{(D)}$ described in [18]. The degeneracy can be described by the two Casimir functions

$$
E_{1}:=d_{0} \quad \text { and } \quad E_{2}:=\left(\prod_{n=0}^{N^{\prime}} c_{n}\right)^{\frac{1}{N^{\prime}+1}}
$$


Let

$$
\mathcal{M}_{\delta, \gamma}^{(D)}:=\left\{(d, c) \in \mathcal{M}^{(D)}:\left(E_{1}, E_{2}\right)=(\delta, \gamma)\right\}
$$

denote the level set of $\left(E_{1}, E_{2}\right)$ for $(\delta, \gamma) \in \mathbb{R} \times \mathbb{R}_{>0}$. Please note that we are mainly interested in the case $\delta=0$, i.e., the set $\mathcal{M}_{0, \gamma}^{(D)}$, because the assumption $\delta \neq 0$ contradicts the boundary conditions (4). We have included the case of general $\delta^{\prime}$ s in order to have an even-dimensional phase space. In the sequel, we write $H_{\delta, \gamma}^{(D)}=\left.H^{(D)}\right|_{\mathcal{M}_{\delta, \gamma}^{(D)}}$.

In [18] we constructed global Birkhoff coordinates for the Dirichlet lattice. Precisely, we constructed a map

$$
\Phi^{(D)}:\left(\mathcal{M}^{(D)}, J^{(D)}\right) \rightarrow\left(\mathcal{P}^{(D)}, J_{0}^{(D)}\right),(d, c) \mapsto\left(\left(x_{n}, y_{n}\right)_{1 \leq n \leq N^{\prime}}, E_{1}, E_{2}\right),
$$

where $E_{1}$ and $E_{2}$ are the Casimir functions introduced by (12). The crucial point of the map (13) is that the coordinates $\left(x_{n}, y_{n}\right)_{1 \leq n \leq N^{\prime}}, E_{1}, E_{2}$ are global Birkhoff coordinates for the Dirichlet Toda lattice, i.e., the transformed Toda Hamiltonian $\hat{H}^{(D)}=H^{(D)} \circ\left(\Omega^{(D)}\right)^{-1}$ is a function of the actions $\left(x_{n}^{2}+y_{n}^{2}\right) / 2$ $\left(1 \leq n \leq N^{\prime}\right)$ and $E_{1}, E_{2}$ alone.

Coordinates for the periodic lattice In the following, we describe an analogous set-up for the periodic lattice. Although the set-up looks similar to what we just introduced for the Dirichlet lattice, it is not identical to the Dirichlet case, which makes it necessary to be introduced explicitly.

The main tool for the investigation of the periodic lattice are the (noncanonical) coordinates $\left(b_{j}, a_{j}\right)_{j \in \mathbb{Z}}$ introduced by Flaschka [14], analogously to (8),

$$
b_{n}:=-p_{n} \in \mathbb{R}, \quad a_{n}:=\alpha e^{\frac{1}{2}\left(q_{n}-q_{n+1}\right)} \in \mathbb{R}_{>0} \quad(n \in \mathbb{Z}) .
$$

In these coordinates the Hamiltonian $H_{\text {Toda }}$, analogously to (9), takes the simple form

$$
H=\frac{1}{2} \sum_{n=1}^{N} b_{n}^{2}+\sum_{n=1}^{N} a_{n}^{2}
$$

and the equations of motion are, analogously to (10),

$$
\left\{\begin{array}{l}
\dot{b}_{n}=a_{n}^{2}-a_{n-1}^{2} \\
\dot{a}_{n}=\frac{1}{2} a_{n}\left(b_{n+1}-b_{n}\right)
\end{array} \quad(n \in \mathbb{Z}) .\right.
$$

Please note that $\left(b_{n+N}, a_{n+N}\right)=\left(b_{n}, a_{n}\right)$ for any $n \in \mathbb{Z}$, and $\prod_{n=1}^{N} a_{n}=\alpha^{N}$, which means that the sequences $\left(b_{n}\right)_{n \in \mathbb{Z}}$ and $\left(a_{n}\right)_{n \in \mathbb{Z}}$ can be identified with the vectors $\left(b_{n}\right)_{1 \leq n \leq N} \in \mathbb{R}^{N}$ and $\left(a_{n}\right)_{1 \leq n \leq N} \in \mathbb{R}_{>0}^{N}$, respectively.

In [31] we studied the normal form of the system of Equation (16) on the phase space

$$
\mathcal{M}:=\mathbb{R}^{N} \times \mathbb{R}_{>0}^{N}
$$

and the model space $\mathcal{P}:=\mathbb{R}^{2(N-1)} \times \mathbb{R} \times \mathbb{R}_{>0}$. Precisely, we constructed a map

$$
\Phi: \mathcal{M} \rightarrow \mathcal{P}, \quad(b, a) \mapsto\left(\left(x_{n}, y_{n}\right)_{1 \leq n \leq N-1}, C_{1}, C_{2}\right),
$$

where $C_{1}, C_{2}$ are Casimir functions associated with the Poisson structure of the Flaschka coordinates (14). The crucial point of the Birkhoff map (18) is that the coordinates 
$\left(x_{n}, y_{n}\right)_{1 \leq n \leq N-1}, C_{1}, C_{2}$ are global Birkhoff coordinates for the periodic Toda lattice. Hence the Toda Hamiltonian, when expressed in these coordinates, takes the form

$$
H \circ \Phi^{-1}=\frac{N \beta^{2}}{2}+H_{\alpha}(I)
$$

where the term $H_{\alpha}(I)$ is a real analytic function of the action variables $I_{n}:=\frac{x_{n}^{2}+y_{n}^{2}}{2},(1 \leq n \leq N-1)$, and where $\beta, \alpha$ are the values of the Casimirs $C_{1}, C_{2}$.

Note that on an open dense subset $\left\{(b, a) \in \mathcal{M} \mid I_{n}(b, a) \neq 0\right\}$ of the phase space $\mathcal{M}$, the coordinates $\left(x_{n}, y_{n}\right)_{1 \leq n \leq N-1}$ of the Birkhoff map (18) are given in terms of action and angle variables $\left(I_{n}, \theta_{n}\right)_{1 \leq n \leq N-1}$ by

$$
\left(x_{n}, y_{n}\right)=\sqrt{I_{n}}\left(\cos \theta_{n}, \sin \theta_{n}\right) .
$$

Symmetries of the periodic lattice Let $T^{*} \mathbb{R}^{N}$ be endowed with the canonical symplectic structure and consider the linear maps $T, S: T^{*} \mathbb{R}^{N} \rightarrow T^{*} \mathbb{R}^{N}$ given by

$$
\begin{aligned}
T:\left(q_{1}, \ldots, q_{N}, p_{1}, \ldots, p_{N}\right) & \mapsto\left(q_{2}, q_{3}, \ldots, q_{N}, q_{1}, p_{2}, p_{3}, \ldots, p_{N}, p_{1}\right), \\
S:\left(q_{1}, \ldots, q_{N}, p_{1}, \ldots, p_{N}\right) & \mapsto-\left(q_{N-1}, \ldots, q_{1}, q_{N}, p_{N-1}, \ldots, p_{1}, p_{N}\right) ;
\end{aligned}
$$

note that $T$ is the standard shift operator. As already discussed by Rink [32] for arbitrary FPU chains, the maps $T$ and $S$ satisfy the relations $T^{N}=S^{2}=\operatorname{Id}$ and $T S=S T^{-1}$. Moreover, $T$ and $S$ are symplectic maps leaving the Hamiltonian $H_{\text {Toda }}$, given by (1), invariant. The group $G_{H}=\langle T, S\rangle$ (a representation of the $N$-th dihedral group $D_{N}$ ) is the symmetry group of $H_{\text {Toda }}$. In the sequel, we are mainly interested in the symmetry map $S$.

Denote by $\operatorname{Fix}(S)$ the fixed point set of the map $S$ introduced above. Then $\operatorname{Fix}(S)$ is the subset of all elements $(q, p)$ in $T^{*} \mathbb{R}^{N}$ satisfying

$$
\left(q_{n}, p_{n}\right)=-\left(q_{N-n}, p_{N-n}\right) \forall 1 \leq n \leq N-1 \text { and } q_{N}=p_{N}=0 .
$$

In particular, if $N$ is even, on $\operatorname{Fix}(S)$ we have $q_{N}=q_{N / 2}=0$ and $p_{N}=p_{N / 2}=0$.

Note that on $\operatorname{Fix}(S)$, both the center of mass coordinate $Q=\frac{1}{N} \sum_{i=1}^{N} q_{i}$ and its momentum $P=\frac{1}{N} \sum_{i=1}^{N} p_{i}$ are identically zero. Hence $\operatorname{Fix}(S) \subseteq\left\{(q, p) \in T^{*} \mathbb{R}^{N} \mid Q=0 ; P=0\right\}$.

On the level of the Flaschka variables $\left(b_{j}, a_{j}\right)_{1 \leq j \leq N}$ introduced in (14), the maps $T$ and $S$ introduced in (21) and (22) are given by $\tilde{T}, \tilde{S}: \mathcal{M} \rightarrow \mathcal{M}$, with $\tilde{S}(b, a) \equiv(\tilde{S}(b), \tilde{S}(a))$ (analogously for $\tilde{T}$ ) and

$$
\begin{array}{rrr}
(\tilde{T}(b))_{j}=b_{j+1}, \quad(\tilde{T}(a))_{j}=a_{j+1}, & (1 \leq j \leq N), \\
(\tilde{S}(b))_{j}=-b_{N-j}, \quad(\tilde{S}(a))_{j}=a_{N-j-1} . & (1 \leq j \leq N) .
\end{array}
$$

Similarly to $\operatorname{Fix}(S)$ defined above, we denote by $\operatorname{Fix}(\tilde{S})$ the subset of all elements $(b, a) \in \mathcal{M}$ satisfying

$$
\left(b_{N-j}, a_{N-j}\right)=\left(-b_{j}, a_{j+1}\right) \text { for any } 1 \leq j \leq N .
$$

with the indices in (24)-(26) understood $\bmod N$. In the sequel, we will omit the tilde and write $T$ and $S$ for the operators $\tilde{T}$ and $\tilde{S}$ on $\mathcal{M}$.

We can also express the symmetry transformations $T$ and $S$ in terms of the Cartesian coordinates $\left(x_{n}, y_{n}\right)_{1 \leq n \leq N-1}$ given by the Birkhoff map (18), or more suitably, in terms of the associated complex coordinates $\left(\zeta_{k}\right)_{1 \leq|k| \leq N-1}$, defined for $1 \leq k \leq N-1$ by

$$
\left\{\begin{aligned}
\zeta_{k} & =\frac{1}{\sqrt{2}}\left(x_{k}-i y_{k}\right) \\
\zeta_{-k}=\overline{\zeta_{k}} & =\frac{1}{\sqrt{2}}\left(x_{k}+i y_{k}\right) .
\end{aligned}\right.
$$


We denote by $z$ the linear subspace of $\mathbb{C}^{2 N-2}$ consisting of such vectors $\left(\zeta_{k}\right)_{1 \leq|k| \leq N-1}$, and define the map

$$
S_{z}: Z \rightarrow Z,\left(\zeta_{k}\right)_{1 \leq|k| \leq N-1} \mapsto\left(-e^{4 \pi i k / N} \zeta_{N-k}\right)_{1 \leq|k| \leq N-1} .
$$

Like the map $S: \mathcal{M} \rightarrow \mathcal{M}$, this new map $S_{z}$ is a linear involution. In fact, the two maps $S$ and $S_{z}$ are conjugate to each other under the Birkhoff map $\Phi$ given by (18)—see [18] for a proof of the following theorem:

Theorem 5. In terms of the complex variables $\left(\zeta_{k}\right)_{1 \leq|k| \leq N-1}$ defined by (27) and the Birkhoff map $\Phi$ given by (18) the map $S$ is given by $S_{z}$. More precisely,

$$
S_{z} \circ \Phi=\Phi \circ S
$$

Embedding of the Dirichlet into the periodic lattice We now embed the phase space $\mathcal{M}^{(D)}$ of the Dirichlet lattice, with $N^{\prime}$ particles, see (11), into the phase space $\mathcal{M}$ of the periodic lattice with $N=2 N^{\prime}+2$ particles, see (17), by the map

$$
\begin{aligned}
& \Theta^{(D)}: \mathcal{M}^{(D)} \rightarrow \mathcal{M} \\
& (d, c)=\left(d_{j}, c_{j}\right)_{0 \leq j \leq N^{\prime}} \mapsto \Theta(d, c)=\left(b_{j}, a_{j}\right)_{1 \leq j \leq N}
\end{aligned}
$$

where

$$
b_{j}=\frac{1}{\sqrt{2}}\left\{\begin{array}{l}
d_{j} \\
0 \\
-d_{N-j} \\
0
\end{array}, \quad a_{j}=\frac{1}{\sqrt{2}}\left\{\begin{array}{ll}
c_{j} & \left(1 \leq j \leq N^{\prime}\right) \\
c_{N^{\prime}} & \left(j=N^{\prime}+1\right) \\
c_{N-j-1} & \left(N^{\prime}+2 \leq j \leq 2 N^{\prime}+1\right) \\
c_{0} & \left(j=2 N^{\prime}+2\right)
\end{array} .\right.\right.
$$

The image of the map $\Theta^{(D)}$ is a subset of $\operatorname{Fix}(S)$, as the following shows (see [18]):

Lemma 1. Let $(\delta, \gamma) \in \mathbb{R} \times \mathbb{R}_{>0}$.

(i) $\Theta^{(D)}\left(\mathcal{M}_{\delta, \gamma}^{(D)}\right)=\mathcal{M}_{0, \frac{\gamma}{\sqrt{2}}} \cap$ Fix $(S)$; in particular, $\Theta^{(D)}(d, c) \in$ Fix $(S)$ for any $(d, c) \in \mathcal{M}^{(D)}$. Moreover, $\Theta_{\delta, \gamma}^{(D)}$ is a canonical map from $\left(\mathcal{M}_{\delta, \gamma}^{(D)}, J^{(D)}\right)$ to $\left(\mathcal{M}_{0, \frac{\gamma}{\sqrt{2}}}, J\right)$.

(ii) The Hamiltonians $H$ and $H^{(D)}$ of the Toda lattice in Flaschka variables with periodic and Dirichlet boundary conditions, respectively, given by (15) and (9), satisfy $H \circ \Theta^{(D)}=H^{(D)}$.

To pull back the embedding $\Theta^{(D)}$ to the complex variables $\left(\zeta_{k}\right)_{1 \leq|k| \leq N-1}$ defined by (27), we consider the space

$$
z^{(D)}:=\left\{\left(\zeta_{k}\right)_{1 \leq|k| \leq N^{\prime}} \in \mathbb{C}^{2 N^{\prime}} \mid \bar{\zeta}_{k}=\zeta_{-k} \quad \forall 1 \leq k \leq N^{\prime}\right\}
$$

endowed with the canonical symplectic structure induced from $\mathbb{C}^{2 N^{\prime}}$. Now consider the embedding

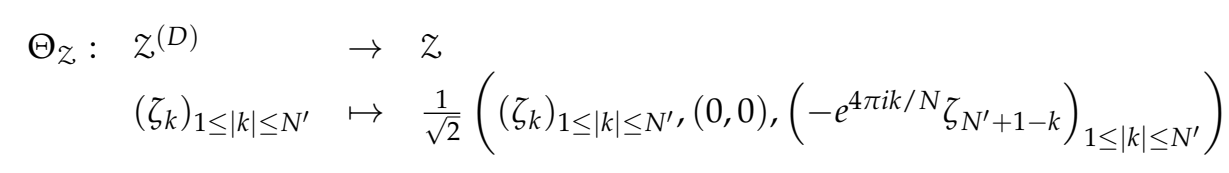

Note that

$$
\Theta_{z}\left(z^{(D)}\right)=\operatorname{Fix}\left(S_{z}\right)
$$


i.e., $\Theta_{z}$ is a parametrization of $\operatorname{Fix}\left(S_{z}\right)$. In other words, by (i), for any $(\delta, \gamma) \in \mathbb{R} \times \mathbb{R}_{>0}$, $\left.\Theta^{(D)}\right|_{\mathcal{M}_{\delta, \gamma}^{(D)}}=: \Theta_{\delta, \gamma}^{(D)}$ is a parametrization of $\mathcal{M}_{0, \frac{\gamma}{\sqrt{2}}} \cap \operatorname{Fix}(S)$. Together with the Birkhoff maps of the periodic and the Dirichlet lattice, this leaves us with the following commutative diagram of maps:

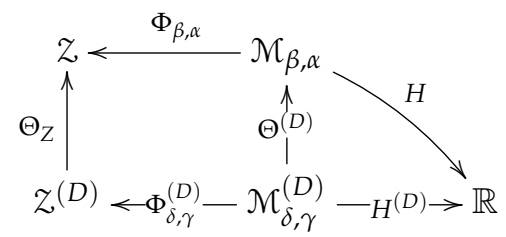

\section{Spectral Quantities and Riemann Surfaces}

It is known (cf. e.g., [33]) that the periodic system (16) can be expressed in the Lax pair form $\dot{L}=\frac{\partial L}{\partial t}=[B, L]$, where $L \equiv L^{+}(b, a)$ is the periodic Jacobi matrix defined by

$$
L^{ \pm}(b, a):=\left(\begin{array}{ccccc}
b_{1} & a_{1} & 0 & \ldots & \pm a_{N} \\
a_{1} & b_{2} & a_{2} & \ddots & \vdots \\
0 & a_{2} & b_{3} & \ddots & 0 \\
\vdots & \ddots & \ddots & \ddots & a_{N-1} \\
\pm a_{N} & \ldots & 0 & a_{N-1} & b_{N}
\end{array}\right)
$$

and a skew-symmetric matrix $B$. Hence the flow of $\dot{L}=[B, L]$ is isospectral.

Discriminant: We denote by $\Delta(\lambda) \equiv \Delta(\lambda, b, a)$ the discriminant of the difference equation

$$
\left(\left(a_{k-1} T^{-1}+b_{k} T^{0}+a_{k} T^{1}\right) y\right)(k)=\lambda y(k) \quad(k \in \mathbb{Z})
$$

associated with the matrix $L$, defined by

$$
\Delta(\lambda):=y_{1}(N, \lambda)+y_{2}(N+1, \lambda),
$$

where $y_{1}(\cdot, \lambda)$ and $y_{2}(\cdot, \lambda)$ are two standard fundamental solutions of (36). In the sequel, we will often write $\Delta_{\lambda}$ for $\Delta(\lambda)$. Additionally, we consider the symmetric $2 N \times 2 N$-Jacobi matrix

$$
Q \equiv Q(b, a)=L((b, b),(a, a))
$$

whose $2 N$ real eigenvalues $\left(\lambda_{j}\right)_{1 \leq j \leq 2 N}$ (with multiplicities) are the zeroes of the function $\Delta_{\lambda}^{2}-4$,

$$
\Delta_{\lambda}^{2}-4=\alpha^{-2 N} \prod_{j=1}^{2 N}\left(\lambda-\lambda_{j}\right) .
$$

(see [34] for comments on this expansion). When listed in increasing order and with their algebraic multiplicities, they fulfill the following relations (cf. [35])

$$
\lambda_{1}<\lambda_{2} \leq \lambda_{3}<\lambda_{4} \leq \lambda_{5}<\ldots \lambda_{2 N-2} \leq \lambda_{2 N-1}<\lambda_{2 N} .
$$

In [18] we proved the following results on the behaviour of the $\lambda_{j}$ 's and the discriminant $\Delta_{\lambda}$ under the symmetry $S$ introduced in (22):

Lemma 2. Let $(b, a) \in \mathcal{M}$. Then for any $1 \leq j \leq 2 N$,

$$
\lambda_{j}(S(b, a))=-\lambda_{2 N+1-j}(b, a) .
$$


Corollary 2. For any $(b, a) \in \operatorname{Fix}(S)$,

(i) $I_{k}(b, a)=I_{N-k}(b, a)$ for any $1 \leq k \leq N-1$.

(ii) $\lambda_{j}(b, a)=-\lambda_{2 N+1-j}(b, a)$ for any $1 \leq j \leq 2 N$.

(iii) $I_{\frac{N}{2}}(b, a)=0$ and $\lambda_{N}(b, a)=\lambda_{N+1}(b, a)=0$, if $N$ is even.

Corollary 3. For any $(b, a) \in \mathcal{M}$,

$$
\Delta_{\lambda}(S(b, a)) \mp 2=(-1)^{N} \Delta_{-\lambda}(b, a) \mp 2,
$$

and hence, with $\dot{\Delta}_{\lambda}=\partial_{\lambda} \Delta_{\lambda}$,

$$
\dot{\Delta}_{\lambda}(S(b, a))=(-1)^{N+1} \dot{\Delta}_{-\lambda}(b, a)
$$

as well as

$$
\Delta_{\lambda}^{2}(S(b, a))-4=\Delta_{-\lambda}^{2}(b, a)-4 .
$$

Riemann surface $\Sigma_{b, a}$ : Denote by $\Sigma_{b, a}$ the Riemann surface obtained as the compactification of the affine curve

$$
\mathfrak{C}_{b, a}:=\left\{(\lambda, z) \in \mathbb{C}^{2}: z^{2}=R(\lambda)\right\}
$$

for

$$
R(\lambda):=\Delta_{\lambda}^{2}(b, a)-4=\alpha^{-2 N} \prod_{j=1}^{2 N}\left(\lambda-\lambda_{j}(b, a)\right)
$$

by (38). Note that $\mathcal{C}_{b, a}$ is a two-sheeted curve with the ramification points $\left(\lambda_{i}, 0\right)_{1 \leq i \leq 2 N}$, identified with $\lambda_{i}$ in the sequel, and that $\mathcal{C}_{b, a}$ and $\Sigma_{b, a}$ are spectral invariants; the Riemann surface $\Sigma_{b, a}$ is obtained from $\mathcal{C}_{b, a}$ by adding two (unramified) points at infinity, namely $\infty^{+}$and $\infty^{-}$, one on each of the two sheets, i.e.,

$$
\Sigma_{b, a}:=\mathcal{C}_{b, a} \cup\left\{\infty^{+}, \infty^{-}\right\} .
$$

Strictly speaking, $\Sigma_{b, a}$ is a Riemann surface only in the case that the spectrum of $Q(b, a)$ is simple, i.e., if the estimates in (39) are strict. We showed in [34] that $I_{n}(b, a)=0$ iff $\lambda_{2 n}(b, a)=\lambda_{2 n+1}(b, a)$, therefore the spectrum of $Q(b, a)$ is simple iff $(b, a) \in \mathcal{M}^{\bullet}$, where

$$
\mathcal{M}^{\bullet}=\left\{(b, a) \in \mathcal{M} \mid I_{n} \neq 0 \quad \forall 1 \leq n \leq N-1\right\} .
$$

If the spectrum of $Q(b, a)$ is not simple, $\Sigma(b, a)$ can be transformed into a Riemann surface by doubling the multiple eigenvalues-see e.g., Section 2 of [36]. We will discuss this case in detail below, see Section 5 , since it is of great importance for the main task of this paper.

Canonical sheet and canonical root: For $(b, a) \in \mathcal{M} \cdot \bullet$ the canonical sheet of $\Sigma_{b, a}$ is given by the set of points $\left(\lambda, \sqrt[c]{\Delta_{\lambda}^{2}-4}\right)$ in $\mathcal{C}_{b, a}$, where the special $c$-root $\sqrt[c]{\Delta_{\lambda}^{2}-4}$ is defined on $\mathbb{C} \backslash \bigcup_{n=0}^{N}\left(\lambda_{2 n}, \lambda_{2 n+1}\right)$ (with $\lambda_{0}:=-\infty$ and $\lambda_{2 N+1}:=\infty$ ) and determined by imposing the sign condition

$$
-i \sqrt[c]{\Delta_{\lambda}^{2}-4}>0 \text { for } \lambda_{2 N-1}<\lambda<\lambda_{2 N}
$$

As a consequence we have for any $1 \leq n \leq N$

$$
\operatorname{sign} \sqrt[c]{\Delta_{\lambda-i 0}^{2}-4}=(-1)^{N+n-1} \text { for } \quad \lambda_{2 n}<\lambda<\lambda_{2 n+1} .
$$

Cycles on $\Sigma_{b, a}$ : We introduce the projection $\pi \equiv \pi_{b, a}: \mathcal{C}_{b, a} \rightarrow \mathbb{C}$ onto the $\lambda$-plane, i.e., $\pi_{b, a}(\lambda, w)=\lambda$ and its extension to a map $\pi_{b, a}: \Sigma_{b, a} \rightarrow \mathbb{C} \cup\{\infty\}$, where $\pi_{b, a}\left(\infty^{ \pm}\right)=\infty$.

We now introduce the cycles $\left(c_{k}\right)_{1 \leq k \leq N-1}$ and $\left(d_{k}\right)_{1 \leq k \leq N-1}$ on $\Sigma_{b, a}$. Denote by $\left(c_{k}\right)_{1 \leq k \leq N-1}$ the cycles on the canonical sheet of $\mathcal{C}_{b, a}$ (see above) in such a way that $\pi\left(c_{k}\right)$ is a counterclockwise oriented 
closed curve in $\mathbb{C}$ which contains in its interior the two ramification points $\lambda_{2 k}$ and $\lambda_{2 k+1}$, whereas all other ramification points are located outside of $\pi\left(c_{k}\right)$.

Moreover, denote by $\left(d_{k}\right)_{1 \leq k \leq N-1}$ pairwise disjoint cycles on $\mathcal{C}_{b, a} \backslash\left\{\left(\lambda_{k}\right)_{1 \leq k \leq N-1}\right\}$ defined in a way such that for any $1 \leq n, k \leq N-1$, the intersection indices with the cycles $\left(c_{n}\right)_{1 \leq n \leq N-1}$ defined above with respect to the orientation on $\Sigma_{b, a}$, induced by the complex structure, are $c_{n} \circ d_{k}=\delta_{n k}$. In order to be more precise, we choose the cycles $d_{k}$ in a way that (i) the projection $\pi_{b, a}\left(d_{k}\right)$ of $d_{k}$ is a smooth and convex counterclockwise oriented curve in $\mathbb{C} \backslash\left(\left(\lambda_{1}, \lambda_{2 k}\right) \cup\left(\lambda_{2 k+1}, \infty\right)\right)$ and that (ii) those points of $d_{k}$ whose projection by $\pi_{b, a}$ onto the $\lambda$-plane have a negative imaginary part are located on the canonical sheet of $\Sigma_{b, a}$.

Abelian differentials: Let $(b, a) \in \mathcal{M} \bullet$ and $1 \leq n \leq N-1$. Then there exists a uniquely given polynomial $\psi_{n}(\lambda)$ of degree at most $N-2$ such that for any $1 \leq k \leq N-1$

$$
\frac{1}{2 \pi} \int_{\mathcal{c}_{k}} \frac{\psi_{n}(\lambda)}{\sqrt{\Delta_{\lambda}^{2}-4}} d \lambda=\delta_{k n} .
$$

Using the definition of the cycles $c_{k}$ given above, we can rewrite (50) as

$$
\frac{1}{\pi} \int_{\lambda_{2 k}}^{\lambda_{2 k+1}} \frac{\psi_{n}(\lambda)}{\sqrt[c]{\Delta_{\lambda-i 0}^{2}-4}} d \lambda=\delta_{k n} .
$$

The coefficients of the polynomials $\psi_{n}(\lambda)$ can be computed explicitly, see e.g., Appendix A of [37]. In [18] we proved

Lemma 3. Let $(b, a) \in \mathcal{M} \bullet$. Then for any real $\lambda$,

$$
\psi_{n}(\lambda)(S(b, a))=(-1)^{N} \psi_{N-n}(-\lambda)(b, a) .
$$

Note that the Formula (63) remains valid if the assumption $(b, a) \in \mathcal{M}^{\bullet}$ is weakened, as long as the $\psi$-functions appearing in (63) are well-defined. We will return to this point in the next section.

On the surface $\Sigma_{b, a}$, we consider the differentials

$$
\begin{aligned}
& \Omega_{1}=-\frac{1}{N} \frac{\dot{\Delta}_{\lambda}}{\sqrt{\Delta_{\lambda}^{2}-4}} d \lambda \\
& \Omega_{2}=-\frac{1}{N}\left(\frac{\lambda \dot{\Delta}_{\lambda}}{\sqrt{\Delta_{\lambda}^{2}-4}} d \lambda-\sum_{n=1}^{N-1} I_{n} \frac{\psi_{n}(\lambda)}{\sqrt{\Delta_{\lambda}^{2}-4}} d \lambda\right) .
\end{aligned}
$$

We proved in [21] the following lemma on $\Omega_{1}$ and $\Omega_{2}$ :

Lemma 4. The Abelian differentials $\Omega_{1}$ and $\Omega_{2}$ on $\Sigma_{b, a}$ satisfy the following properties:

(i) $\Omega_{1}$ and $\Omega_{2}$ are holomorphic differentials on $\Sigma_{b, a}$ except at the points $f \infty^{+}$and $\infty^{-}$where in the standard charts, the $\Omega_{i}$ 's admit an expansion of the following form

$$
\Omega_{1}=\mp\left(\frac{1}{\lambda}-\frac{e_{1}}{\lambda^{2}}+O\left(\frac{1}{\lambda^{3}}\right)\right) d \lambda, \quad \Omega_{2}=\mp\left(1+O\left(\frac{1}{\lambda^{2}}\right)\right) d \lambda .
$$

(ii) $\Omega_{1}$ and $\Omega_{2}$ fulfill the normalization condtions

$$
\int_{\mathcal{c}_{k}} \Omega_{i}=0 \quad \forall 1 \leq k \leq N-1, i=1,2 .
$$


(iii) When expressed in the local coordinate $\lambda$, on each of the two sheets, $\int_{\lambda_{2 N}}^{\lambda} \Omega_{1}$ has an asymptotic expansion as $\lambda \rightarrow \infty$ ( $\lambda$ real) of the following form

$$
\int_{\lambda_{2 N}}^{\lambda} \Omega_{1}=\mp\left(\log \lambda+e_{0}+e_{1} \frac{1}{\lambda}+\ldots\right) .
$$

On $\Sigma_{b, a} \backslash\left\{\lambda_{1}, \ldots, \lambda_{2 N}\right\}, \Omega_{1}$ and $\Omega_{2}$ take the form

$$
\Omega_{i}=\frac{\chi_{i}(\lambda)}{\sqrt{R(\lambda)}} d \lambda \quad(i=1,2)
$$

where $\chi_{i}(\lambda)$ are polynomials in $\lambda$ of the form $\chi_{1}(\lambda)=\lambda^{N-1}+e \lambda^{N-2}+\ldots$ and $\chi_{2}(\lambda)=\lambda^{N}+f \lambda^{N-1}+\ldots$. Note that $\Omega_{1}$ and $\Omega_{2}$ do not depend on the parameter $\alpha$. Conversely, (i) and (ii) uniquely determine $\Omega_{1}$ and $\Omega_{2}$.

Remark 1. As discussed in [21], the differentials $\Omega_{1}$ and $\Omega_{2}$ exist for any strictly increasing sequence of $\lambda_{j}$ 's, i.e., $\lambda_{1}<\ldots<\lambda_{2 N}$, even if these $\lambda_{j}$ 's are not the spectrum of some matrix $Q(b, a)$. In this more general case, the associated Riemann surface, similarly to (44)-(46), is defined as the compactification of

$$
\mathcal{C}:=\left\{(\lambda, z) \in \mathbb{C}^{2}: z^{2}=\alpha^{-2 N} \prod_{j=1}^{2 N}\left(\lambda-\lambda_{j}\right)\right\}
$$

i.e.,

$$
\Sigma=\mathcal{C} \cup\left\{\infty^{+}, \infty^{-}\right\}
$$

The differentials $\Omega_{1}$ and $\Omega_{2}$ are then simply defined by the conditions (55) and (56) instead of the Formulas (53) and (54).

For any $1 \leq k \leq N-1$, introduce the $d_{k}$-periods of the differentials $\Omega_{1}$ and $\Omega_{2}$,

$$
U_{k}:=\int_{d_{k}} \Omega_{1} ; \quad V_{k}:=\int_{d_{k}} \Omega_{2} .
$$

In [21], we proved the following results on the $U_{k}{ }^{\prime} \mathrm{s}$, the $V_{k}$ 's and $e_{0}$, the coefficient in the expansion (57); recall from (19) that $H_{\alpha}$ is the Toda Hamiltonian expressed in the Birkhoff coordinates given by (18), $H \circ \Phi^{-1}=\frac{N \beta^{2}}{2}+H_{\alpha}(I)$ :

Proposition 1. For any $(b, a) \in \mathcal{M} \bullet$ and any $1 \leq k \leq N-1$,

$$
U_{k}=\frac{2 \pi i k}{N}, \quad V_{k}=\frac{2}{i} \omega_{k}, \quad e_{0}=-\log (\alpha) .
$$

where $\omega_{k}$ is the Toda frequency $\omega_{k}=\partial_{I_{k}} H_{\alpha}$.

\section{Constructions on the Fixed Point Set}

We now assume that $N \geq 6$ is even, i.e., $N=2 N^{\prime}+2$ for some $N^{\prime} \geq 2$. In addition to $\operatorname{Fix}(S)$, we consider the smaller set (analogously to $\mathcal{M}^{\bullet}$ )

$$
\mathcal{M}_{\text {Fix }}^{\bullet}=\left\{(b, a) \in \operatorname{Fix}(S) \subseteq \mathcal{M} \mid I_{k}(b, a)>0 \quad \forall 1 \leq k \leq N^{\prime}\right\} .
$$

It follows from Corollary (2) (ii) that in the case $(b, a) \in \mathcal{M}_{F i x}^{\bullet}$ the spectrum of $Q(b, a)$ is not simple, hence $\Sigma_{b, a}$, as defined by (44) and (46), is not a Riemann surface in this case, but rather a "punctured" surface (a neighborhood of the point $\left(\lambda_{N}, 0\right)$ is a bouquet of two open disks glued together at one common point, hence the surface does not have a manifold strucutre at this point-see [36]). We can overcome this difficulty by the following construction. 
Instead of $R(\lambda)$ as defined by (45), we consider

$$
R^{(r e g)}(\lambda):=\alpha^{-2 N+2} \prod_{\substack{1 \leq k \leq 2 N \\ k \neq N, N+1}}\left(\lambda-\lambda_{k}\right)
$$

and the surface

$$
\Sigma_{b, a}^{(r e g)}:=\left\{(\lambda, z) \in \mathbb{C}^{2}: z^{2}=R^{(r e g)}(\lambda)\right\} \cup\left\{\infty^{+}, \infty^{-}\right\} .
$$

Note that unlike in the definition of $R_{b, a}$, see (45), $R^{(r e g)}(\lambda) \neq \Delta_{\lambda}^{2}(b, a)-4$. For $(b, a) \in \mathcal{M}_{\text {Fix }}^{\bullet}$, the surface $\Sigma_{b, a}^{(\text {reg })}$ is isomorphic to the regular Riemann surface $\Sigma_{b, a}^{*}$ obtained from the punctured surface $\Sigma_{b, a}$ by doubling the point $\left(\lambda_{N}(b, a), 0\right)$, as shown in [36] for the analogous case of finite gap potentials of the KdV equation. In the sequel, we will thus consider $\Sigma_{b, a}^{*}$ instead of $\Sigma_{b, a}^{(r e g)}$. Note that the genus of $\Sigma_{b, a}^{*}$ equals the genus of $\Sigma_{b, a}$ minus one.

The cycles $\left(c_{k}\right)_{1 \leq k \leq N-1}$ and $\left(d_{k}\right)_{1 \leq k \leq N-1}$, defined in Section 4 for $\Sigma_{b, a}$, are defined on $\Sigma_{b, a}^{*}$ as well, as long as $k \neq \frac{N}{2}$. In the case $(b, a) \in \mathcal{M}_{F i x}^{\bullet}$, the curve on $\Sigma_{b, a}^{*}$ corresponding to $c_{N / 2}$ does not contain any ramification point in its interior, and the curve on $\Sigma_{b, a}^{*}$ corresponding to $d_{N / 2}$ is no longer closed . On $\Sigma_{b, a^{\prime}}^{*}$ we thus consider the $2(N-2)$ curves $\left(c_{k}\right)_{1 \leq k \leq N-1, k \neq N / 2}$ and $\left(d_{k}\right)_{1 \leq k \leq N-1, k \neq N / 2}$.

On $\Sigma_{b, a^{\prime}}^{*}$ the functions $\psi_{n}(\lambda)$, defined by (50), exist as well, as long as $n \neq \frac{N}{2}$, since there existence only depends on the cycles $c_{n}$. Lemma 3 now takes the form

Lemma 5. Let $(b, a) \in \mathcal{M}_{\text {Fix }}^{\bullet}$. Then for any real $\lambda$,

$$
\psi_{n}(\lambda)(b, a)=(-1)^{N} \psi_{N-n}(-\lambda)(b, a) .
$$

The proof of Lemma 5 works completely along the lines of the proof of Lemma 3, which we have given in [18] and which is based on Lemma 2 (which does not assume $(b, a) \in \mathcal{M}^{\bullet}$ ), and we then use $(b, a)=S(b, a)$. We will use (63) in Appendix B in the proof of Lemma 6 below.

Moreover, we again consider the differentials $\Omega_{1}$ and $\Omega_{2}$ defined by (53) and (54), now on $\Sigma_{b, a}^{*}$; for clarity, we momentarily denote these differentials on the new surface $\Sigma_{b, a}^{*}$ by

$$
\begin{aligned}
& \Omega_{1}^{*}=-\frac{1}{N} \frac{\dot{\Delta}_{\lambda}}{\sqrt{\Delta_{\lambda}^{2}-4}} d \lambda, \\
& \Omega_{2}^{*}=-\frac{1}{N}\left(\frac{\lambda \dot{\Delta}_{\lambda}}{\sqrt{\Delta_{\lambda}^{2}-4}} d \lambda-\sum_{\substack{n=1 \\
n \neq \frac{N}{2}}}^{N-1} I_{n} \frac{\psi_{n}(\lambda)}{\sqrt{\Delta_{\lambda}^{2}-4}} d \lambda\right) .
\end{aligned}
$$

They have the same properties as described by Lemma 4 for the original case $(b, a) \in \mathcal{M}^{\bullet}$, except that the identities (56) are only valid for $k \neq \frac{N}{2}$ and $\Sigma_{b, a}$ has to be replaced by $\Sigma_{b, a}^{*}$. That these properties continue to hold in this new case $(b, a) \in \mathcal{M}_{F i x}^{\bullet}$ follows from the fact that the surfaces $\Sigma_{b, a}$ and $\Sigma_{b, a}^{*}$ differ only in the fact that the point $\left(\lambda_{n}, 0\right)$ has been doubled in the construction of $\Sigma_{b, a^{\prime}}^{*}$ and the curves $\left(c_{k}\right)_{1 \leq k \leq N-1}$ for $k \neq \frac{N}{2}$ do not touch this doubled point.

Remark 1 also holds in this case: The Riemann surface $\Sigma^{*}$ and the differentials $\Omega_{1}^{*}$ and $\Omega_{2}^{*}$ exist for any sequence of $\lambda^{\prime}$ s with $\lambda_{1}<\lambda_{2}<\ldots<\lambda_{N}=\lambda_{N+1}<\ldots \lambda_{2 N}$, even if this sequence is not the spectrum of $Q(b, a)$ for some $(b, a) \in \mathcal{M}_{F i x}^{\bullet}$. Namely, $\Sigma^{*}$ is in such a case defined by, similarly to (58), as the compactification of the affine curve

$$
\mathcal{C} \equiv \mathcal{C}_{\lambda}:=\left\{(\lambda, z) \in \mathbb{C}^{2}: z^{2}=\alpha^{-2 N} \prod_{\substack{1 \leq j \leq 2 N \\ k \neq N, N+1}}\left(\lambda-\lambda_{j}\right)\right\}
$$


i.e., as

$$
\Sigma^{*} \equiv \Sigma_{\lambda}^{*}=\mathcal{C}_{\lambda} \cup\left\{\infty^{+}, \infty^{-}\right\}
$$

The differentials $\Omega_{1}^{*}$ and $\Omega_{2}^{*}$ are then defined by the properties (55) and (56), where it is understood that (56) only holds for $k \neq \frac{N}{2}$. For clarity, we restate this definition in Appendix C, see Lemma A1. If the sequence $\lambda_{1}<\lambda_{2}<\ldots<\lambda_{N}=\lambda_{N+1}<\ldots \lambda_{2 N}$ corresponds to some $(b, a) \in \mathcal{M}_{F i x}^{\bullet}$, one shows that the differentials defined by (64) and (65) have the properties (55) and (56), and in the general case the existence and uniqueness of differentials with these properties is established by general Riemann surface theory. All these facts can be checked exactly as in the case discussed in the previous section, and in our previous work [21].

For $k \neq \frac{N}{2}$, we consider as in the original case (59) the $d_{k}$-periods of $\Omega_{1}^{*}$ and $\Omega_{2}^{*}$, with the integrals now taken on the new surface $\Sigma^{*}$, defined by (67),

$$
U_{k}^{*}:=\int_{d_{k}} \Omega_{1}^{*} ; \quad V_{k}^{*}:=\int_{d_{k}} \Omega_{2}^{*} \quad\left(1 \leq k \leq N-1, k \neq \frac{N}{2}\right) .
$$

On these $U_{k}^{* \prime}$ s and $V_{k}^{* \prime}$ in the case $(b, a) \in \mathcal{M}_{F i x}^{\bullet}$, we have the following lemma, which we prove in Appendix B.

Lemma 6. Let $(b, a) \in \mathcal{M}_{\text {Fix }}^{\bullet}$. Then for any $1 \leq k<\frac{N}{2}$,

$$
\begin{aligned}
U_{N-k}^{*} & =-U_{k}^{*}+2 \pi i, \\
V_{N-k}^{*} & =V_{k}^{*} .
\end{aligned}
$$

for any $1 \leq k<\frac{N}{2}$.

Note that we only claim the statements (69) and (70) to be true in the case of a Riemann surface $\Sigma_{b, a}^{*}$ originating from a sequence of $\lambda_{j}$ 's being the spectrum of $Q(b, a)$ for some $(b, a) \in \mathcal{M}_{F i x}^{\bullet}$ and not in the case of an arbitrary surface $\Sigma^{*}$; but we only need the formulas in the former (more special) case, see the proof of Theorem 6 .

We now define the extended period map $\mathcal{F}$ on the set

$$
\mathcal{L}:=\left\{\lambda=\left(\lambda_{j}\right)_{1 \leq j \leq 2 N^{\prime}+1} \in \mathbb{R}^{2 N^{\prime}+1} \mid \lambda_{1}<\ldots<\lambda_{2 N^{\prime}+1}<0\right\}
$$

as follows

$$
\begin{aligned}
\mathcal{F}: \mathcal{L} & \rightarrow \mathbb{R}^{2 N^{\prime}+1} \\
\lambda & \mapsto\left(\left(U_{i}^{*}, V_{i}^{*}\right)_{1 \leq i \leq N^{\prime}}, e_{0}\right),
\end{aligned}
$$

where $e_{0}$ is the coefficient in the asymptotic expansion (57). In the construction (71), $\lambda \in \mathcal{L}$ is extended to $\tilde{\lambda}=(\lambda, 0,0,-\lambda) \in \mathbb{R}^{2 N}$. To this auxiliary $\tilde{\lambda}$, the associated surface $\Sigma^{*}$ is constructed via (66) and (67), on which the differentials $\Omega_{1}^{*}$ and $\Omega_{2}^{*}$ are given, and then the quantities $U_{k}^{*}$ and $V_{k}^{*}$ by (68). It is straightforward to see that $\mathcal{F}$ is a smooth map with values in $\mathbb{R}^{2 N^{\prime}+1}$.

Next we define the map

$$
\begin{aligned}
\Lambda: \quad \mathbb{R}_{>0}^{N^{\prime}} \times \mathbb{R}_{>0} & \rightarrow \mathcal{L} \\
\left(\left(I_{n}\right)_{1 \leq n \leq N^{\prime}}, \alpha\right) & \mapsto\left(\lambda_{j}\right)_{1 \leq j \leq 2 N^{\prime}+1}
\end{aligned}
$$

where $\left(\lambda_{n}\right)_{1 \leq n \leq 2 N^{\prime}+1}$ is the first part of the spectrum of the matrix $Q(b, a)$ for some $(b, a) \in \mathcal{M}_{F i x}^{\bullet}$ which is determined by the Birkhoff map $\Phi$ (cf. (18)),

$$
(b, a)=\Phi^{-1}\left(\left(\sqrt[+]{2 I_{n}}, 0\right)_{1 \leq n \leq N^{\prime}},(0,0),\left(\sqrt[+]{2 I_{N^{\prime}+1-n}}, 0\right)_{1 \leq n \leq N^{\prime}}, 0, \alpha\right)
$$


Note that (73) ensures that $(b, a) \in \mathcal{M}_{\text {Fix }}^{\bullet}$ and therefore $\lambda \in \mathcal{L}$. It can be seen analogously as in the case $(b, a) \in \mathcal{M} \bullet$ explained in [21] that $\Lambda$ is a smooth embedding.

Proposition 2. For any $\lambda=\left(\lambda_{1}<\ldots<\lambda_{2 N^{\prime}+1}\right) \in \mathcal{L}$,

$$
\mathcal{F}(\lambda)=\left(\left(\frac{2 n \pi i}{N},-2 i \partial_{I_{n}} H_{\alpha}\right)_{1 \leq n \leq N^{\prime}},-\log \alpha\right) .
$$

Proof. Recall from Proposition 1 that in the case $(b, a) \in \mathcal{M}^{\bullet}$, we have

$$
U_{k}=\frac{2 \pi i k}{N}, \quad V_{k}=\frac{2}{i} \omega_{k} \quad(1 \leq k \leq N)
$$

and $\omega_{k}=\partial_{I_{n}} H_{\alpha}$ by definition. In our case $(b, a) \in \mathcal{M} \mathcal{C}^{\bullet}$, these formulas continue to hold for the $U_{k}^{* \prime} \mathrm{s}$ and $V_{k}^{* \prime}$, as long as $k \neq \frac{N}{2}$, and their proof is completely analogous to the proof of the former case discussed in our previous work [21]. We will explicitly show the case of the $U_{k}$ 's in the proof of Lemma 6 in Appendix B. From the Formula (75) and the definition (71) of the map F, the claimed statement (74) immediately follows.

The composition $\mathcal{F} \circ \Lambda: \mathbb{R}_{>0}^{N^{\prime}} \times \mathbb{R}_{>0} \rightarrow \mathbb{R}^{2 N^{\prime}+1}$ is therefore given by

$$
\mathcal{F} \circ \Lambda\left(\left(I_{n}\right)_{1 \leq n \leq N^{\prime}}, \alpha\right)=\left(\left(\frac{2 \pi n i}{N},-2 i \partial_{I_{n}} H_{\alpha}\right)_{1 \leq n \leq N^{\prime}},-\log \alpha\right) .
$$

The differential $d(\mathcal{F} \circ \Lambda)$ is a $\left(2 N^{\prime}+1\right) \times\left(N^{\prime}+1\right)$-matrix of the form

$$
\left(\begin{array}{c|c}
0_{N^{\prime} \times N^{\prime}} & 0_{N^{\prime} \times 1} \\
\hline\left(-2 i \frac{\partial^{2} H_{\alpha}}{\partial_{I_{n}} \partial_{I_{l}}}\right)_{1 \leq n, l \leq N^{\prime}} & 0_{N^{\prime} \times 1} \\
\hline \cdots & -\alpha^{-1}
\end{array}\right),
$$

where $0_{N_{1} \times N_{2}}$ denotes the $N_{1} \times N_{2}$-matrix with all entries 0 . We now need the following version of Krichever's theorem $[21,38]$ on the map $\mathcal{F}$. Note that the following theorem is only concerned with the behaviour of $\mathcal{F}$ on $\operatorname{Im}(\Lambda)$, the image of the map $\Lambda$ as defined by (72).

Theorem 6. Restricted to $\operatorname{Im}(\Lambda)$, the map $\mathcal{F}$ is a local embedding, i.e., the differential

$$
d_{\lambda}\left(\left.\mathcal{F}\right|_{I m(\Lambda)}\right): T_{\lambda} \operatorname{Im}(\Lambda) \cong \mathbb{R}^{N^{\prime}+1} \rightarrow T_{\mathcal{F}(\lambda)} \mathbb{R}^{2 N^{\prime}+1} \cong \mathbb{R}^{2 N^{\prime}+1}
$$

of $\mathcal{F}$ at $\lambda$ is a linear embedding.

Proof. Let $\lambda \in \operatorname{Im}(\Lambda) \subseteq \mathcal{L}$, and assume that Theorem 6 does not hold, i.e., that $\left.\mathcal{F}\right|_{\operatorname{Im}(\Lambda)}$ is not a local embedding. Then there exists a smooth 1-parameter family

$$
\lambda(\tau)=\left(\lambda_{j}(\tau)\right)_{1 \leq j \leq 2 N^{\prime}+1}, \quad-1<\tau<1,
$$

with $\lambda(0)=\lambda$ and $\lambda(\tau) \in \operatorname{Im}(\Lambda) \subseteq \mathcal{L}$ for any $-1<\tau<1$, so that

$$
\left.\delta \lambda_{n} \equiv \partial_{\tau}\right|_{\tau=0} \lambda_{n}(\tau) \neq 0 \quad \text { for some } \quad 1 \leq n \leq 2 N^{\prime}+1
$$


but

$$
\left(\left(U_{i}, V_{i}\right)_{1 \leq i \leq N^{\prime}}, e_{0}\right)(\tau)=\left(\left(U_{i}, V_{i}\right)_{1 \leq i \leq N^{\prime}}, e_{0}\right)(0)+O\left(\tau^{2}\right) .
$$

We extend the family $\lambda(\tau)$ with $2 N^{\prime}+1$ components to a family

$$
\lambda(\tau)=\left(\lambda_{n}(\tau)\right)_{1 \leq n \leq 2 N}
$$

with $2 N=4 N^{\prime}+4$ components by

$$
\lambda_{2 N+1-n}(\tau):=-\lambda_{n}(\tau) \quad(1 \leq n \leq N-1), \quad \lambda_{N}(\tau)=\lambda_{N+1}(\tau):=0 .
$$

By this construction, it is ensured that the family $\lambda(\tau)=\left(\lambda_{n}(\tau)\right)_{1 \leq n \leq 2 N}$ obtained in this way is the full spectrum of a matrix $Q(b(\tau), a(\tau))$ for any $-1<\tau<1$, since any element $\lambda=\left(\lambda_{j}\right)_{1 \leq j \leq 2 N^{\prime}+1} \in \operatorname{Im}(\Lambda) \subseteq \mathcal{L}$ is by definition the first part of the spectrum of a matrix $Q(b, a)$ for some $(b, a) \in \mathcal{M}_{F i x}^{\bullet}$. In the sequel, by $\lambda(\tau)$ we mean the $2 N$-component family (80) whose first $2 N^{\prime}+1$ components are given by (77) and whose remaining $2 N^{\prime}+3$ components by (81).

Then, by Lemma 6, (79) remains satisfied, but for the $N-2$ component vectors $U=\left(U_{1}, \ldots, U_{N^{\prime}}, U_{N^{\prime}+2}, \ldots, U_{N-1}\right)$ and analogously for the $V_{k}$ 's. i.e., we have

$$
\begin{aligned}
U_{N-k}(\tau) & =-U_{k}(\tau)+2 \pi i \\
& =-U_{k}(0)+2 \pi i+O\left(\tau^{2}\right) \\
& =U_{N-k}(0)+O\left(\tau^{2}\right),
\end{aligned}
$$

and analogously for the $V_{k}$ 's. Moreover, we have $e_{1}(\tau)=e_{1}(0)=0$, since (as we showed in [21]), $e_{1}=-\beta$, and as we discussed in [18], $\beta=-\frac{1}{N} \sum_{j=1}^{N} b_{j}=-\frac{1}{2 N} \sum_{j=1}^{2 N} \lambda_{j}$. By the conditions (81) however, the last sum equals zero. Alltogether, we obtain

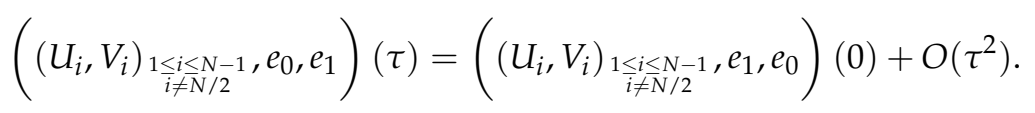

Lemma 7. From the estimates (82) it follows that

$$
\delta \lambda_{n}=0 \quad \text { for any } 1 \leq n \leq N-1, n \neq \frac{N}{2} .
$$

The proof of Theorem 6 is completed once we have proved Lemma 7, since the conclusion (83) obviously contradicts the assumption (78). The proof of Lemma 7 is contained in Appendix C; it completely follows the lines of a similar proof in our previous work [21].

Since both $\Lambda$ and $\mathcal{F} \mid \operatorname{Im}(\Lambda)$ are embeddings, the same holds for the composition $\mathcal{F} \circ \Lambda$. Hence the rank of the differential $d(\mathcal{F} \circ \Lambda)$, given by (76), has to be maximal, i.e., $N^{\prime}+1$. Therefore, by the structure of the matrix (76), the rank of the $N^{\prime} \times N^{\prime}$-matrix $\left(\partial^{2} H_{\alpha} / \partial I_{n} \partial I_{l}\right)_{1 \leq n, l \leq N^{\prime}}$ has to be $N^{\prime}$. We have thus proved the following result on the Hamiltonian of the $N$-particle periodic lattice with respect to the first $N^{\prime}$ action variables in the case $(b, a) \in \mathcal{M}_{F i x}^{\bullet}$ :

Proposition 3. Let $(b, a) \in \mathcal{M}_{\text {Fix }}^{\bullet}$. Then the matrix

$$
\left(\frac{\partial^{2} H_{\alpha}}{\partial I_{n} \partial I_{l}}\right)_{1 \leq n, l \leq N^{\prime}}
$$

is regular. 
We now prove our main result, Theorem 3. The main ingredients are Proposition 3 and the embedding of the $N^{\prime}$-particle Dirichlet lattice into the $2 N^{\prime}+2$-particle periodic lattice described in Section 3.

Proof of Theorem 3. By Proposition 3, the $N^{\prime} \times N^{\prime}$-matrix (84) is regular for any $(b, a) \in \mathcal{M}_{\text {Fix }}^{\bullet}$. Taking the pullback with respect to the embedding $\Theta_{Z}$ given by (32), we have from the commutative diagram (34) the identity

$$
H_{\alpha} \circ \Theta_{Z}=H \circ \Phi_{\beta, \alpha}^{-1} \circ \Theta_{Z}=H^{(D)} \circ\left(\Phi_{\delta, \gamma}^{(D)}\right)^{-1},
$$

and it therefore follows from Proposition 3 that the matrix

$$
\left(\frac{\partial^{2}\left(H^{(D)} \circ\left(\Phi_{\delta, \gamma}^{(D)}\right)^{-1}\right)}{\partial I_{n} \partial I_{l}}\right)_{1 \leq n, l \leq N^{\prime}}
$$

is regular as well (note that the property of being a nondegenerate matrix is invariant under coordinate transformations). In other words, the frequency map

$$
\mathbb{R}_{\geq 0}^{N^{\prime}} \rightarrow \mathbb{R}^{N^{\prime}}, I \mapsto \omega(I ; \gamma):=\partial_{I} \mathcal{H}_{\gamma} .
$$

is nondegenerate on all of $\mathbb{R}_{>0}^{N^{\prime}}$. Together with the convexity of $\mathcal{H}_{\gamma}(I)$ at $I=0$, an immediate consequence of Corollary 1 , this implies that $\mathcal{H}_{\gamma}(I)$ is convex on all of $\mathbb{R}_{>0}^{N^{\prime}}$. This proves Theorem 3.

\section{Discussion}

As already stated in the introduction, establishing a Nekhoroshev-type result for the Toda lattice with Dirichlet boundary conditions in our view has a double significance. On the one hand, it is an interesting statement in the context of the unexpected stability results discovered by Fermi et al. for the general class of FPU chains, in particular for this kind of boundary conditions which is closer to Fermi's simulations and possible physical applications than periodic boundary conditions. On the other hand, our result it is one of the few explicit case studies of the Nekhoroshev theorem; in particular, explicitly checking that the rather strong conditions of the abstract Nekhoroshev theorem are fulfilled is a highly nontrivial task which for many physically interesting examples turns out to be impossible.

Funding: This research was supported by a private fellowship.

Acknowledgments: It is a pleasure to thank Thomas Kappeler for valuable discussions. In addition, the author is grateful for the helpful comments of the reviewers.

Conflicts of Interest: The author declares no conflict of interest. The funding source had no role in the design of the study, in the writing of the manuscript, and in the decision to publish the result.

\section{Appendix A. Nekhoroshev's Theorem}

Here we describe the theoretical set-up of perturbed Hamiltonian systems and Nekhoroshev's theorem on the stability of the motion in such systems, thereby exactly following the exposition in [20]. In this reference, the statement is formulated for convex and quasi-convex unperturbed Hamiltonians; we only cite the version for the (stronger) convex case, since it is this version which we apply in the present paper.

We consider a neary integrable Hamiltonian

$$
H=H_{0}(I)+f_{\epsilon}(I, \theta),
$$


which is a real analytic function of the action-angle variables $I \in R \subseteq \mathbb{R}^{n}, \theta \in \mathbb{T}^{n}=\mathbb{R}^{n} / 2 \pi \mathbb{Z}^{n}$ and arbitrarily depends on a small parameter $\epsilon$ (we assume $n \geq 2$ ). Specifically, the Hamiltonian is assumed to be a real analytic function on a fixed complex neighborhood of $P \times \mathbb{T}^{n}$ of the form $V_{r_{0}, s_{0}} P=V_{r_{0}} P \times W_{s_{0}} \mathbb{T}^{n} \subseteq \mathbb{C}^{n} \times \mathbb{C}^{n}$. The Hessian of the integrable Hamiltonian $H_{0}, Q(I)=\partial_{I}^{2} H_{0}(I)$, is assumed to be uniformly bounded with respect to the operator norm induced by the Euclidean norm.

We assume the perturbation parameter $\epsilon$ to be chosen in such a way that $\left|f_{\epsilon}\right|_{P, s_{0}, r_{0}} \leq \epsilon$ in the following exponentially weighted norm: If $u$ is analytic on $V_{r, s} D$ with a Fourier expansion $\sum_{k} u_{k}(I) e^{i k \cdot \theta}$, then

$$
\left|u_{\epsilon}\right|_{P, s_{0}, r_{0}}=\sup I \in V_{r}(D) \sum_{k \in \mathbb{Z}^{n}}\left|u_{k}(I)\right| e^{|k| s},
$$

where we use the 1-norm $|k|=\left|k_{1}\right|+\ldots+\left|k_{n}\right|$.

Let $m$ be a positive number. The integrable Hamiltonian $H_{0}$ is denoted $m$-convex, if the inequality

$$
\langle Q(I) \xi, \xi\rangle \geq m\|\xi\|^{2}, \quad \xi \in \mathbb{R}^{n},
$$

holds at every point $I \in U_{r_{0}} P$. Nekhoroshev's theorem on the exponential stability of the motion governed by the perturbed Hamiltonian (A1) then reads as follows:

Theorem A1. Suppose $H_{0}$ is m-convex, and

$$
\left|f_{\epsilon}\right|_{P, s_{0}, r_{0}} \leq \epsilon \leq \epsilon_{0}=\frac{m r_{0}^{2}}{2^{10} A^{2 n}}
$$

where $r_{0} \leq 4 l / m$ and $A=11 \mathrm{M} / \mathrm{m}$. Then for every orbit with initial position $\left(I_{0}, \theta_{0}\right) \in P \times \mathbb{T}^{n}$ one has the estimate

$$
\left\|I(t)-I_{0}\right\| \leq R_{0}\left(\frac{\epsilon}{\epsilon_{0}}\right)^{a} \quad \text { for } \quad|t| \leq T_{0} \exp \left(\frac{s_{0}}{6}\left({\frac{\epsilon_{0}}{\epsilon}}^{a}\right)\right)
$$

except when $\left\|\omega\left(I_{0}\right)\right\| \leq m r_{0} / 8$ in which case $\left\|I(t)-I_{0}\right\| \leq r_{0}$ for all $t$. The parameters are

$$
a=\frac{1}{2 n}, \quad R_{0}=\frac{r_{0}}{A}, \quad T_{0}=A^{2} \frac{s_{0}}{\Omega_{0}},
$$

where $\Omega_{0}=\sup _{\left\|I-I_{0}\right\| \leq R_{0}}\|\omega(I)\|$.

\section{Appendix B. Additional Properties of the Period Map}

Here we prove Lemma 6 on the behaviour of the $d_{n}$-periods $U_{k}^{*}$ and $V_{k}^{*}$ of the differentials $\Omega_{1}^{*}$ and $\Omega_{2}^{*}$ on the set $\mathcal{M}_{F i x}^{*}$, defined by (60). In this appendix, we omit the stars and write $U_{k}, V_{k}, \Omega_{1}$ and $\Omega_{2}$.

Proof of Lemma 6. Since the Riemann surfaces $\Sigma_{b, a}$ in the case $(b, a) \in \mathcal{M} \bullet$ and $\Sigma_{b, a}^{*}$ in the case $(b, a) \in \mathcal{M}_{F i x}^{\bullet}$ are topologically different (in particular, they have a different genus), we prove the claimed Formulas (69) and (70) by direct computations and without refering to formulas in the case $(b, a) \in \mathcal{M} \mathfrak{M}^{\bullet}$ and continuity arguments. The computations are however similar to the case $(b, a) \in \mathcal{M} \bullet$; in particular, the sign conditions for the canonical sheet and the canonical root $\sqrt[c]{\Delta_{\lambda}^{2}-4}$ on the surface $\Sigma_{b, a}^{*}$ remain unchanged, since on the complex plane $\mathbb{C}$, the behaviour of the sign of $\sqrt{\Delta_{\lambda}^{2}-4}$ for $(b, a) \in \mathcal{M}_{F i x}^{\bullet}$ is qualitatively similar to the corresponding behaviour in the case $(b, a) \in \mathcal{M}^{\bullet}$; the spectral gap $\left[\lambda_{N}, \lambda_{N+1}\right]$ in the latter case has simply shrinked to a single point in the former case.

We first prove the statement (69) on the $U_{k}$ 's, closely following the corresponding computation in [21]. By the definition (64) and the normalization conditions (56) one gets for any $1 \leq n \leq N-1$ with $n \neq \frac{N}{2}$

$$
\int_{d_{n}} \Omega_{1}=-\frac{2}{N}\left(\int_{\lambda_{1}}^{\lambda_{2}}+\int_{\lambda_{3}}^{\lambda_{4}}+\ldots+\int_{\lambda_{2 n-1}}^{\lambda_{2 n}}\right) \frac{\dot{\Delta}_{\lambda}}{\sqrt[c]{\Delta_{\lambda-i 0}^{2}-4}} d \lambda .
$$


For any $\lambda_{2 k-1} \leq \lambda \leq \lambda_{2 k}$, by the sign condition (49),

$$
\frac{\dot{\Delta}_{\lambda}}{\sqrt[c]{\Delta_{\lambda-i 0}^{2}-4}}=\frac{(-1)^{N-k} \dot{\Delta} / 2}{i+\sqrt{1-(\Delta / 2)^{2}}} d \lambda=\frac{1}{i} \partial_{\lambda}\left(\arcsin \left((-1)^{N-k} \frac{\Delta_{\lambda}}{2}\right)\right)
$$

and thus

$$
\int_{d_{n}} \Omega_{1}=-\left.\frac{2}{N i} \sum_{k=1}^{n} \arcsin \left((-1)^{N-k} \frac{\Delta_{\lambda}}{2}\right)\right|_{\lambda_{2 k-1}} ^{\lambda_{2 k}}=\frac{2 n \pi}{N} i .
$$

Using Formula (A2) for $n=N-k$, we get

$$
U_{N-k}=\frac{2(N-k) \pi}{N} i=2 \pi i-\frac{2 k \pi i}{N}=2 \pi i-U_{k}
$$

as claimed.

We now turn to the statement (70) on the $V_{k}$ 's. By the normalization conditions (56), we get

$$
V_{n}=\int_{d_{n}} \Omega_{2}=2 \cdot\left(\int_{\lambda_{1}}^{\lambda_{2}}+\ldots+\int_{\lambda_{2 n-1}}^{\lambda_{2 n}}\right) \Omega_{2}
$$

By (54), $\Omega_{2}$ is given by

$$
\Omega_{2}=-\frac{1}{N}\left(\frac{\lambda \dot{\Delta}_{\lambda}}{\sqrt{\Delta_{\lambda}^{2}-4}} d \lambda-\sum_{n=1}^{N-1} I_{n} \frac{\psi_{n}(\lambda)}{\sqrt{\Delta_{\lambda}^{2}-4}} d \lambda\right)
$$

Hence it follows that (using the normalization conditions (56) for $1 \leq n<\frac{N}{2}$ )

$$
\begin{aligned}
V_{n} & =\int_{d_{n}} \Omega_{2}=-\frac{1}{N}\left(\int_{d_{n}} \frac{\lambda \dot{\Delta}_{\lambda}}{\sqrt{\Delta_{\lambda}^{2}-4}} d \lambda-\sum_{l=1}^{N-1} I_{l} \int_{d_{n}} \frac{\psi_{l}(\lambda)}{\sqrt{\Delta_{\lambda}^{2}-4}} d \lambda\right) . \\
& =-\frac{2}{N}(\sum_{k=1}^{n} \underbrace{\int_{\lambda_{2 k-1}}^{\lambda_{2 k}} \frac{\lambda \dot{\Delta}_{\lambda}}{\sqrt[c]{\Delta_{\lambda-i 0}^{2}-4}} d \lambda}_{=: A_{k}}-\sum_{l=1}^{N-1} I_{l} \sum_{k=1}^{n} \underbrace{\int_{\lambda_{2 k-1}}^{\lambda_{2 k}} \frac{\psi_{l}(\lambda)}{\sqrt[c]{\Delta_{\lambda-i 0}^{2}-4}} d \lambda}_{B_{k, l}})
\end{aligned}
$$

Using the notation with the $A_{k}$ 's and $B_{k, l}$ 's just introduced, we get for $1 \leq n<\frac{N}{2}$

$$
\begin{aligned}
V_{N-n} & =-\frac{2}{N}\left(\sum_{k=1}^{N-n} A_{k}-\sum_{l=1}^{N-1} I_{l} \sum_{k=1}^{N-n} B_{k, l}\right) \\
& =-\frac{2}{N}\left(\left(\sum_{k=1}^{n}+\sum_{k=n+1}^{N-n}\right) A_{k}-\sum_{l=1}^{N-1} I_{l}\left(\sum_{k=1}^{n}+\sum_{k=n+1}^{N-n}\right) B_{k, l}\right) \\
& =V_{n}-\frac{2}{N}\left(\sum_{k=n+1}^{N-n} A_{k}-\sum_{l=1}^{N-1} I_{l} \sum_{k=n+1}^{N-n} B_{k, l}\right)
\end{aligned}
$$

We claim that we have for any $1 \leq k, l \leq N-1$

$$
\begin{aligned}
A_{k} & =-A_{N+1-k} \\
B_{k, l} & =-B_{N+1-k, N-l} .
\end{aligned}
$$


Before proving (A4) and (A5), we finish the proof of (70). By (A4), it follows that

$$
\begin{aligned}
\sum_{k=n+1}^{N-n} A_{k} & =\frac{1}{2}\left(\sum_{k=n+1}^{N-n} A_{k}-\sum_{k=n+1}^{N-n} A_{N+1-k}\right) \\
& =\frac{1}{2}\left(\sum_{k=n+1}^{N-n} A_{k}-\sum_{m=n+1}^{N-n} A_{m}\right)=0,
\end{aligned}
$$

where we made the substitution $m=N+1-k$ in the second sum. Similarly, we use (A5) to obtain

$$
\begin{aligned}
\sum_{l=1}^{N-1} I_{l} \sum_{k=n+1}^{N-n} B_{k, l} & =\frac{1}{2}\left(\sum_{l=1}^{N-1} I_{l} \sum_{k=n+1}^{N-n} B_{k, l}-\sum_{l=1}^{N-1} I_{l} \sum_{k=n+1}^{N-n} B_{N+1-k, N-l}\right) \\
& =\frac{1}{2}\left(\sum_{l=1}^{N-1} I_{l} \sum_{k=n+1}^{N-n} B_{k, l}-\sum_{l=1}^{N-1} I_{N-l} \sum_{k=n+1}^{N-n} B_{N+1-k, N-l}\right) \\
& =\frac{1}{2}\left(\sum_{l=1}^{N-1} I_{l} \sum_{k=n+1}^{N-n} B_{k, l}-\sum_{l=1}^{N-1} I_{N-l} \sum_{m=n+1}^{N-n} B_{m, N-l}\right) \\
& =\frac{1}{2}\left(\sum_{l=1}^{N-1} I_{l} \sum_{k=n+1}^{N-n} B_{k, l}-\sum_{l^{\prime}=1}^{N-1} I_{l^{\prime}} \sum_{m=n+1}^{N-n} B_{m, l^{\prime}}\right) \\
& =0
\end{aligned}
$$

where we used the identity $I_{l}=I_{N-l}$ valid on $\operatorname{Fix}(S)$ (see Corollary 2) and then the substitutions $m=N+1-k$ and $l^{\prime}=N-l$. Plugging (A6) and (A8) into (A4), we get $V_{N-n}=V_{n}$, i.e., the claimed Formula (70).

It remains to prove the auxiliary Formulas (A4) and (A5). We first turn to (A4). By the sign rule (49) for the $c$-root we get

$$
\begin{aligned}
A_{n} & =\int_{\lambda_{2 n-1}}^{\lambda_{2 n}} \frac{\lambda \dot{\Delta}_{\lambda}}{\sqrt[c]{\Delta_{\lambda-i 0}^{2}-4}} d \lambda \\
& =(-1)^{N-n} \int_{\lambda_{2 n-1}}^{\lambda_{2 n}} \frac{\lambda \dot{\Delta}_{\lambda}}{i \sqrt[+]{4-\Delta_{\lambda}^{2}}} d \lambda
\end{aligned}
$$

By (40), we have $\lambda_{j}=-\lambda_{2 N+1-j}$ on $\operatorname{Fix}(S)$, hence we obtain

$$
\begin{aligned}
A_{n} & =(-1)^{N-n} \int_{-\lambda_{2 N+2-2 n}}^{-\lambda_{2 N+1-2 n}} \frac{\lambda \dot{\Delta}_{\lambda}}{i \sqrt[+]{4-\Delta_{\lambda}^{2}}} d \lambda \\
& =(-1)^{N-n} \cdot(-1) \cdot \int_{\lambda_{2 N+2-2 n}}^{\lambda_{2 N+1-2 n}} \frac{(-\mu) \dot{\Delta}_{-\mu}}{i \sqrt{4-\Delta_{-\mu}^{2}}} d \mu \\
& =(-1)^{n} \cdot \int_{\lambda_{2 N+1-2 n}}^{\lambda_{2 N+2-2 n}} \frac{\mu \dot{\Delta}_{\mu}}{i \sqrt{4-\Delta_{\mu}^{2}}} d \mu
\end{aligned}
$$

in the second step making the substitution $\mu=-\lambda$ and in the last step reversing the integration direction and using Corollary 3 in the last step; recall that $N=2 N^{\prime}+2$ is even, which implies

$$
\dot{\Delta}_{-\mu}=(-1)^{N+1} \dot{\Delta}_{\mu}=-\dot{\Delta}_{\mu}
$$


and simplifies the sign in front of the integral. Again writing $\lambda=\mu$ and using the rule (49) for the sign of the $c$-root, we get

$$
\begin{aligned}
A_{n} & =(-1)^{n} \cdot \int_{\lambda_{2 N+1-2 n}}^{\lambda_{2 N+2-2 n}} \frac{\lambda \dot{\Delta}_{\lambda}}{i \sqrt[+]{4-\Delta_{\lambda}^{2}}} d \lambda \\
& =(-1)^{n} \cdot(-1) \int_{\lambda_{2(N+1-n)-1}}^{\lambda_{2(N+1-n)}} \frac{\lambda \dot{\Delta}_{\lambda}}{(-1)^{N-(N+1-n)} \sqrt[c]{\Delta_{\lambda-i 0}^{2}-4}} d \lambda \\
& =-\int_{\lambda_{2(N+1-n)-1}}^{\lambda_{2(N+1-n)}} \frac{\lambda \dot{\Delta}_{\lambda}}{\sqrt[c]{\Delta_{\lambda-i 0}^{2}-4}} d \lambda \\
& =-A_{N-k}
\end{aligned}
$$

as claimed in (A4). The proof of (A5) proceeds in a similar way, and we mainly comment on the differences to the previous computation. We have, using the same steps as above,

$$
\begin{aligned}
B_{n, l} & =\int_{\lambda_{2 k-1}}^{\lambda_{2 k}} \frac{\psi_{l}(\lambda)}{\sqrt[c]{\Delta_{\lambda-i 0}^{2}-4}} d \lambda \\
& =(-1)^{N-n} \int_{\lambda_{2 n-1}}^{\lambda_{2 n}} \frac{\psi_{l}(\lambda)}{i \sqrt[+]{4-\Delta_{\lambda}^{2}}} d \lambda \\
& =(-1)^{N-n} \int_{\lambda_{2 N+1-2 n}}^{\lambda_{2 N+2-2 n}} \frac{\psi_{l}(-\lambda)}{i \sqrt[+]{4-\Delta_{\lambda}^{2}}} d \lambda
\end{aligned}
$$

Now we use the Formula (63) from Lemma 5. This gives us

$$
B_{n, l}=(-1)^{n} \int_{\lambda_{2 N+1-2 n}}^{\lambda_{2 N+2-2 n}} \frac{\psi_{N-l}(\lambda)}{i \sqrt[+]{4-\Delta_{\lambda}^{2}}} d \lambda .
$$

As before, we then get

$$
\begin{aligned}
B_{n, l} & =(-1)^{n} \int_{\lambda_{2 N+1-2 n}}^{\lambda_{2 N+2-2 n}} \frac{\psi_{N-l}(\lambda)}{(-1)^{N-(N+1-n)} \sqrt[c]{\Delta_{\lambda}^{2}-4}} d \lambda \\
& =-\int_{\lambda_{2(N+1-n)-1}}^{\lambda_{2(N+1-n)}} \frac{\psi_{N-l}(\lambda)}{c} d \lambda \\
& =-B_{N+1-n, N-l},
\end{aligned}
$$

as claimed in (A4). This completes the proof of Lemma 6.

\section{Appendix C. Proof of the Modified Bikbaev-Kuksin Lemma}

Note that this entire appendix is basically copied from our previous work [21], with the necessary small modifications due to the fact that we are considering a slightly different Riemann surface than in the situation discussed in [21].

First we need to derive some auxiliary results. Let us first recall Lemma 4, now reformulated for our purpose of the Riemann surface $\Sigma^{*}$ defined by (67), and regardless of whether the sequence of $\lambda^{\prime} s$ under consideration is a part of the spectrum of some $(b, a) \in \mathcal{M}_{\text {Fix }}^{\bullet}$ :

Lemma A1. There exist Abelian differentials $\Omega_{1}^{*}$ and $\Omega_{2}^{*}$ on $\Sigma^{*}$ uniquely defined by the the following properties: 
(i) $\Omega_{1}^{*}$ and $\Omega_{2}^{*}$ are holomorphic differentials on $\Sigma^{*}$ except at the points $\infty^{+}$and $\infty^{-}$where in the standard charts, the $\Omega_{i}^{*}$ 's admit an expansion of the following form

$$
\Omega_{1}^{*}=\mp\left(\frac{1}{\lambda}-\frac{e_{1}}{\lambda^{2}}+O\left(\frac{1}{\lambda^{3}}\right)\right) d \lambda, \quad \Omega_{2}^{*}=\mp\left(1+O\left(\frac{1}{\lambda^{2}}\right)\right) d \lambda
$$

(ii) $\Omega_{1}^{*}$ and $\Omega_{2}^{*}$ satisfy the normalization condtions

$$
\int_{\mathcal{C}_{k}} \Omega_{i}^{*}=0 \quad \forall 1 \leq k \leq N-1, k \neq \frac{N}{2}, i=1,2 .
$$

(iii) When expressed in the local coordinate $\lambda$, on each of the two sheets, $\int_{\lambda_{2 N}}^{\lambda} \Omega_{1}^{*}$ has an asymptotic expansion as $\lambda \rightarrow \infty$ ( $\lambda$ real $)$ of the following form

$$
\int_{\lambda_{2 N}}^{\lambda} \Omega_{1}^{*}=\mp\left(\log \lambda+e_{0}+e_{1} \frac{1}{\lambda}+\ldots\right) .
$$

On $\Sigma^{*} \backslash\left\{\lambda_{1}, \ldots, \lambda_{2 N}\right\}, \Omega_{1}^{*}$ and $\Omega_{2}^{*}$ take the form

$$
\Omega_{i}^{*}=\frac{\chi_{i}(\lambda)}{\sqrt{R(\lambda)}} d \lambda \quad(i=1,2),
$$

where $\chi_{1}(\lambda)$ and $\chi_{2}(\lambda)$ are polynomials in $\lambda$ of degree $N-1$ and $N-2$, respectively, and

$$
R(\lambda)=\prod_{\substack{1 \leq i \leq 2 N \\ B \neq N, N+1}}\left(\lambda-\lambda_{i}\right)
$$

As in Appendix B, in the sequel we again omit the * and write $\Omega_{1}$ and $\Omega_{2}$, always meaning the differentials $\Omega_{1}^{*}$ and $\Omega_{2}^{*}$ defined by the conditions (A8) and (A9). In addition to these two differentials, for any $p \in \mathcal{C}$ (the affine curve (66) underlying $\Sigma$ ) we define the Abel integrals

$$
J_{i}(p)=\frac{1}{2} \int_{\gamma_{p}} \Omega_{i}
$$

where $\gamma_{p}$ is any path in the set $\mathcal{C}$ from $p_{*}$ to $p$. The map $\iota: \mathcal{C} \rightarrow \mathcal{C}, p \mapsto p_{*}$ interchanges the two sheets of $\mathcal{C}$,

$$
p_{*}=(\lambda,-w) \quad \forall p=(\lambda, w) \in \mathcal{C} .
$$

Note that for any $i=1,2$, the function $p \mapsto J_{i}(p)$ is multi-valued. Actually, $J_{i}(p)$ is well defined up to half periods of $\Omega_{i}$. Therefore locally it is a well defined smooth function. Consequently, its differential $d J_{i}$ is well defined. Note that for $i=1,2$ and $1 \leq n \leq 2 N(n \neq N, N+1)$, the number zero is one of the possible values of $J_{i}\left(\lambda_{n}\right)$. For any point $p \in \mathcal{C}$, we denote by $\gamma_{p}^{0}$ a path in $\mathcal{C}$ from $\lambda_{2 N} \equiv\left(\lambda_{2 N}, 0\right)$ to $p$, and we define $\gamma_{p}$ to be the path from $p_{*}$ to $p$ (see (A13)) obtained by concatenating $-\iota\left(\gamma_{p}^{0}\right)$ and $\gamma_{p}^{0}$. Here the path $-\iota\left(\gamma_{p}^{0}\right)$ denotes the path from $p_{*}$ to $\lambda_{2 N}$ obtained by reversing the orientation of the path $\iota\left(\gamma_{p}^{0}\right)$, and $\iota\left(\gamma_{p}^{0}\right)$ is the path obtained by applying to $\gamma_{p}^{0}$ the map $\iota$. In Lemma A2 we state those properties of $\Omega_{i}$ and $J_{i}$ which we need in the sequel.

Lemma A2. (i) The differential forms $\Omega_{1}$ and $\Omega_{2}$ are odd with respect to the interchanging map l, i.e., the pullback $\iota^{*} \Omega_{i}$ of $\Omega_{i}$ satisfies the identity $\iota^{*} \Omega_{i}=-\Omega_{i}$.

(ii) For $i=1,2$,

$$
\frac{1}{2} \int_{-\iota\left(\gamma_{P}^{0}\right) \circ \gamma_{P}^{0}} \Omega_{i}=\int_{\gamma_{P}^{0}} \Omega_{i} .
$$


(iii) When expressed in the local coordinate $\lambda$, on each of the two sheets, the integral $\int_{\lambda_{2 N}}^{\lambda} \Omega_{i}$ admits an asymptotic expansion as $\lambda \rightarrow \infty$ ( $\lambda$ real) of the following form

$$
\int_{\lambda_{2 N}}^{\lambda} \Omega_{1}=\mp\left(\log \lambda+e_{0}+e_{1} \frac{1}{\lambda}+\ldots\right)
$$

and

$$
\int_{\lambda_{2 N}}^{\lambda} \Omega_{2}=\mp\left(\lambda+f_{0}+\ldots\right)
$$

where $e_{0}$ and $e_{1}$ are real valued.

Proof. (i) Let $1 \leq i \leq 2$. The claimed identity $\iota^{*} \Omega_{i}=-\Omega_{i}$ follows from the uniqueness of the differential $\Omega_{i}$ stated above in Lemma A1, as $-\iota^{*} \Omega_{i}$ is a meromorphic differential which is holomorphic on the surface $\mathcal{C}$ and satisfies the same asymptotics at $\infty^{ \pm}$as well as the same normalization condition (A9) as the differential $\Omega_{i}$.

(ii) In view of statement (i) we conclude that for any point $p \in \mathcal{C}$,

$$
\frac{1}{2} \int_{-\iota\left(\gamma_{P}^{0}\right) \circ \gamma_{P}^{0}} \Omega_{i}=\frac{1}{2}\left(-\int_{\iota\left(\gamma_{P}^{0}\right)} \Omega_{i}+\int_{\gamma_{P}^{0}} \Omega_{i}\right)=\int_{\gamma_{P}^{0}} \Omega_{i}
$$

(iii) The stated asymptotics follow from the asymptotics of $\Omega_{i}$ stated in Lemma A1. The claim that $e_{0}$ and $e_{1}$ are real follows from the assumption that $\lambda_{1}, \ldots, \lambda_{2 N}$ are real and that for any real $\lambda$ with $\lambda>\lambda_{2 N}$, one has $R(\lambda)>0$.

For any $1 \leq i \leq 2$, we denote by $N_{\Omega_{i}}$ the set of zeroes of $\Omega_{i}$, and we denote by $N_{\chi_{i}}$ the set of zeroes of the polynomials $\chi_{i}$, where in both cases the zeroes are listed with their respective multiplicities. Note that we have $\left|N_{\chi_{1}}\right|=N-2$ and $\left|N_{\chi_{2}}\right|=N-1$, whereas for $i=1,2$

$$
\left|N_{\Omega_{i}}\right| \leq 2\left|N_{\chi_{i}}\right| .
$$

We introduce the projection $\pi \equiv \pi_{\lambda}: \mathcal{C} \rightarrow \mathbb{C}$ onto the $\lambda$-plane, i.e., the projection map $\pi(\lambda, w)=\lambda$ and its extension to a map $\pi: \Sigma^{*} \rightarrow \mathbb{C} \cup\{\infty\}$, where $\pi\left(\infty^{ \pm}\right)=\infty$.

Lemma A3. The zero sets $N_{\chi_{i}}$ and $N_{\Omega_{i}}$ have the following properties:

(i) All elements of $N_{\chi_{1}}$ are simple and real, and we have $N_{\chi_{1}} \cap\left\{\lambda_{1}, \ldots, \lambda_{2 N}\right\}=\varnothing$. Moreover, $N_{\Omega_{1}}=\pi_{\lambda}^{-1}\left(N_{\chi_{1}}\right)$ and $\left|N_{\Omega_{1}}\right|=2 N-2$.

(ii) All elements of $N_{\chi_{2}}$ are simple except possibly one which in that case has multiplicity two. Furthermore,

$$
\left|N_{\chi_{2}} \backslash\left\{\lambda_{1}, \ldots, \lambda_{2 N}\right\}\right| \geq N-2 \text { and }\left|N_{\Omega_{2}} \backslash\left\{\lambda_{1}, \ldots, \lambda_{2 N}\right\}\right| \geq 2 N-4 .
$$

(iii) $\quad N_{\chi_{1}} \cap N_{\chi_{2}}=\varnothing$, and thus $N_{\Omega_{1}} \cap N_{\Omega_{2}}=\varnothing$ as well.

Proof. The statements about the zero sets $N_{\Omega_{i}}$ of $\Omega_{i}$ can be easily obtained from the ones about the zero sets $N_{\chi_{i}}$ in view of the representation $\Omega_{i}=\chi_{i}(\lambda) / \sqrt{R(\lambda)} d \lambda$, cf. (A11) above, and the property that $\Omega_{i}$ has a pole at the points $\infty^{+}$and $\infty^{-}$. Hence we only prove the claimed statements for $N_{\chi_{i}}$.

By the normalization condition (A9) above, for any $1 \leq k \leq N-1, k \neq \frac{N}{2}$, the polynomial $\chi_{1}(\lambda)$ has at least one real zero $\tau_{1, k}$ satisfying the condition $\lambda_{2 k}<\tau_{1, k}<\lambda_{2 k+1}$. As $\chi_{1}(\lambda)$ is a polynomial of degree $N-2$, it follows that all zeroes $\tau_{1, k}$ of $\chi(\lambda)$ are simple and that we have

$$
N_{\chi_{1}}=\left\{\tau_{1, k} \mid 1 \leq k \leq N-1, k \neq \frac{N}{2}\right\} .
$$


In particular, note that $N_{\chi_{1}} \cap\left\{\lambda_{1}, \ldots, \lambda_{2 N}\right\}=\varnothing$. Similarly, (A9) implies that for any $1 \leq k \leq N-1, k \neq \frac{N}{2}$, the polynomial $\chi_{2}(\lambda)$ has at least one real zero $\tau_{2, k}$ satisfying the condition $\lambda_{2 k}<\tau_{2, k}<\lambda_{2 k+1}$. As $\chi_{2}(\lambda)$ is a polynomial of degree $N-1$, it follows that

$$
N_{\chi_{2}} \backslash\left\{\tau_{2, k} \mid 1 \leq k \leq N-1, k \neq \frac{N}{2}\right\}
$$

consists of one point $\tau_{0} \in \mathbb{C}$. It is not excluded that this point $\tau_{0}$ coincides with one of the zeroes $\left(\tau_{2, k}\right)_{1 \leq k \leq N-1, k \neq \frac{N}{2}}$. In any case, we have $\left|N_{\chi_{2}} \cap\left\{\lambda_{1}, \ldots, \lambda_{2 N}\right\}\right| \leq 1$. It remains to prove the statement (iii). Assume that a point $\tau$ is a common zero of $\chi_{1}(\lambda)$ and $\chi_{2}(\lambda)$, i.e., that $\tau \in N_{\chi_{1}} \cap N_{\chi_{2}}$. Then there exists $1 \leq k \leq N-1, k \neq \frac{N}{2}$ with $\lambda_{2 k}<\tau<\lambda_{2 k+1}$. As all the roots of $\chi_{1}(\lambda)$ are simple, one has $\chi_{1}^{\prime}(\tau) \neq 0\left({ }^{\prime}=\frac{d}{d \lambda}\right)$. Thus we can choose the real parameter $\xi$ such that the polynomial $\chi_{2}+\xi \chi_{1}$ has a double root at $\tau$. Indeed, for the choice $\xi=-\chi_{2}^{\prime}(\tau) / \chi_{1}^{\prime}(\tau)$ we have $\chi_{2}(\tau)+\xi \chi_{1}(\tau)=0$ and $\chi_{2}^{\prime}(\tau)+\xi \chi_{1}^{\prime}(\tau)=0$. As $\int_{\mathcal{c}_{j}}\left(\chi_{2}(\lambda)+\xi \chi_{1}(\lambda)\right) / \sqrt{R(\lambda)} d \lambda=0$ for all $1 \leq j \leq N-1, j \neq \frac{N}{2}$, the $N$ roots of the polynomial $\chi_{2}+\xi \chi_{1}$ are given by $\tau$ and $\left(\tau_{\xi, j}\right)_{j \neq k}$, where $\tau$ is the double root mentioned above and for any $j \neq k, \frac{N}{2}$, the root $\lambda_{2 j}<\tau_{\zeta, j}<\lambda_{2 j+1}$ is simple. Therefore, the polynomial $\chi_{2}(\lambda)+\xi \chi_{1}(\lambda)$ does not change sign in the interval $\left[\lambda_{2 k}, \lambda_{2 k+1}\right]$, contradicting the normalization condition $\int_{\mathcal{c}_{k}}\left(\chi_{2}(\lambda)+\xi \chi_{1}(\lambda)\right) / R(\lambda) d \lambda=0$. Hence the polynomials $\chi_{1}$ and $\chi_{2}$ have no zero in common, as claimed.

Proof of Lemma 7. The following proof closely imitates the proof of a similar statement in [21], with only slight deviations due to the fact that here we work in the case $\lambda_{N}(\tau)=\lambda_{N+1}(\tau)$. We omit some parts of the proof which are identical to the previously considered case.

For $p \in \mathcal{C}_{\lambda(\tau)}$ the multi-valued functions $J_{i}(p, \tau)$, defined up to half periods of the differentials $\Omega_{i}(\tau)$, are given by

$$
J_{i}(p, \tau)=\frac{1}{2} \int_{\gamma_{p}} \Omega_{i}(\tau)
$$

cf. (A12), where $\Omega_{i}(\tau)$, for $i=1,2$, denote the Abelian differentials of Lemma A1, corresponding to the Riemann surface $\Sigma_{\lambda(\tau)}=\mathcal{C}_{\lambda(\tau)} \cup\left\{\infty^{+}, \infty^{-}\right\}$, as in (66) and (67).

By Lemma A2 (ii), $J_{i}(p, \tau)=\int_{\gamma_{p}^{0}} \Omega_{i}(\tau)$. In particular, note that fpr $i=1,2$ the differential $d J_{i}(p, \tau)$ is well defined and equals the restriction of the differential $\Omega_{i}(\tau)$ to $\mathcal{C}_{\lambda(\tau)}$. Near any point $p=(\lambda, w) \in \mathcal{C} \backslash\left\{\lambda_{1}, \ldots, \lambda_{2 N}\right\}, \lambda$ is a local coordinate. This remains valid for $\tau$ sufficiently close to 0 , and hence for any point $p \in \mathcal{C} \backslash\left\{\lambda_{1}, \ldots, \lambda_{2 N}\right\}$ we can define $(i=1,2)$ as follows:

$$
\delta J_{i}(p):=\left.\partial_{\tau}\right|_{\tau=0} J_{i}(p, \tau) .
$$

By Lemma A4 below, $\delta J_{1}$ is a single-valued function, extends to a meromorphic function on $\Sigma^{*}$ and is holomorphic on the set $\Sigma^{*} \backslash\left\{\lambda_{1}, \ldots, \lambda_{2 N}\right\}$. At a ramification point $\lambda_{k}$, the function $\delta J_{1}$ might have a simple pole with a residue of the form $r_{1}(k) \delta \lambda_{k}$, where $r_{1}(k) \neq 0$. However, by Proposition A1 below, we have $\delta J_{1} \equiv 0$ and hence, in particular, $\delta \lambda_{k}=0$ for any $1 \leq k \leq 2 N$. This alltogether contradicts the assumption made above that $\delta \lambda_{n} \neq 0$. Hence the proof is thus complete once we have proved Lemma A4 and Proposition A1.

Lemma A4. The functions $\delta J_{1}$ and $\delta J_{2}$ defined by (A16) are single-valued functions, and they extend to meromorphic functions on $\Sigma^{*}$. They are holomorphic on the set $\Sigma^{*} \backslash\left\{\lambda_{1}, \ldots, \lambda_{2 N}\right\}$. At the ramification points $\left(\lambda_{n}\right)_{1 \leq n \leq 2 N, n \neq N, N+1}$, they might have simple poles with a residue of the form $(i=1,2 ; 1 \leq n \leq 2 N ; n \neq N, N+1)$

$$
\operatorname{Res}_{p=\lambda_{n}} \delta J_{i}=r_{i}(n) \delta \lambda_{n}
$$

where for $i=1, r_{1}(n) \neq 0$ for any $1 \leq n \leq 2 N, n \neq N, N+1$. In addition, $\delta J_{1}$ has a zero of order 2 at $\infty^{ \pm}$. 
Proof. The proof of this lemma is identical to the proof of Lemma 3.5 in [21]; hence we do not give any details here.

Proposition A1. $\delta J_{1} \equiv 0$.

To prove the statement of Proposition A1, we first need to introduce an auxiliary function. For any point $p \in \mathcal{C} \backslash N_{\Omega_{1}}, d J_{1}(p)=\Omega_{1}(p) \neq 0$. Hence it follows by the implicit function theorem that there exists a smooth curve $\tau \mapsto q(\tau):=q(\tau, p)$ with $q(0)=p$ defined for $\tau$ sufficiently close to zero such that $J_{1}(q(\tau), \tau)=J_{1}(p)$. In particular, one has one has $q(\tau)=\lambda_{n}(\tau)$ for $p=\lambda_{n}(n \neq N, N+1)$. Then we introduce for $p \in \mathcal{C} \backslash N_{\Omega_{1}}$

$$
\delta K(p):=\left.\frac{d}{d \tau}\right|_{\tau=0} J_{2}(q(\tau), \tau)
$$

As the periods of the differentials $\Omega_{2}$ are constant up to $O\left(\tau^{2}\right)$, and as $J_{2}(p, \tau)$ is well defined up to half periods of $\Omega_{2}$, the function $\delta \mathrm{K}$ is single-valued. Moreover, $\delta \mathrm{K}$ admits a meromorphic extension to $\Sigma^{*}$. Indeed, as we have $J_{1}(q(\tau), \tau)=J_{1}(p)$, it follows that for any $p \in \mathcal{C} \backslash N_{\Omega_{1}}$

$$
\delta J_{1}(p)+\left\langle\Omega_{1}(p), \delta q\right\rangle=0
$$

where the pairing $\langle\cdot, \cdot\rangle$ denotes the dual pairing between $T_{p}^{*} \Sigma^{*}$ and $T_{p} \Sigma^{*}$. Therefore

$$
\delta K(p)=\left.\frac{d}{d \tau}\right|_{\tau=0} J_{2}(q(\tau), \tau)=\delta J_{2}(p)+\left\langle\Omega_{2}(p), \delta q\right\rangle
$$

leads to

$$
\delta K(p)=\delta J_{2}(p)-\frac{\Omega_{2}(p)}{\Omega_{1}(p)} \delta J_{1}(p)
$$

By Lemma A3 we know that $\frac{\Omega_{2}(p)}{\Omega_{1}(p)}$ extends to a meromorphic function on $\Sigma^{*}$ with possible poles at the zeroes of $\Omega_{1}$. Because of Lemma A4, $\delta \mathrm{K}$ admits a meromorphic extension to $\Sigma^{*}$.

Lemma A5. $\delta K \equiv 0$.

Proof of Lemma A5. We show that, when counted with their orders, the number of poles of the differential $\delta K$ does not match the number of zeroes. First note that we have $\delta K\left(\lambda_{n}\right)=0$ for any $1 \leq n \leq 2 N, n \neq N, N+1$. Indeed, if $p=\lambda_{n}$ for some $n$ satisfying $1 \leq n \leq 2 N, n \neq N, N+1$, we have $q(\tau)=\lambda_{n}(\tau)$ and therefore for $i=1,2, J_{i}\left(\lambda_{n}(\tau), \tau\right)$ contains zero for any $\tau$, implying that the identity $\delta K\left(\lambda_{n}\right)=0$ holds. On the other hand, by the definition (A17) of $\delta K$, the poles of $\delta K$ in $\mathcal{C}$ are contained in the set $N_{\Omega_{1}}$ of the zeroes of $\Omega_{1}$. By Lemma A3, all those zeroes are simple and hence $\left|N_{\Omega_{1}}\right|=2 N-4$. Now we investigate the values of $\delta K$ at $\infty^{+}$and $\infty^{-}$. Using the standard charts $z=\frac{1}{\lambda}$ it follows by Lemma 4 that

$$
\frac{\Omega_{2}(z)}{\Omega_{1}(z)}=O\left(\frac{1}{z}\right)
$$

and by Lemma A4, we have $\delta J_{1}(z)=O\left(z^{2}\right)$. Therefore

$$
\frac{\Omega_{2}(z)}{\Omega_{1}(z)} \delta J_{1}(z)=O(z)
$$

It follows that $\frac{\Omega_{2}(z)}{\Omega_{1}(z)} \delta J_{1}(z)$ vanishes at $\infty^{+}$and $\infty^{-}$. Moreover, again by Lemma A4, $\delta J_{2}$ is holomorphic at $\infty^{+}$and $\infty^{-}$. Alltogether it follows that the meromorphic function $\delta K$ has at least $2 N-2$ zeroes and at most $2 N-4$ poles (counted with multiplicities). As $\Sigma^{*}$ is a compact surface, it then follows that $\delta K \equiv 0$. 
Proof of Proposition A1. By Lemma A5, Formula (A17) implies that

$$
\delta J_{1} \cdot \Omega_{2} \equiv \delta J_{2} \cdot \Omega_{1} .
$$

By comparing the poles and the zeroes of $\delta J_{2} \cdot \Omega_{1}$ and $\delta J_{1} \cdot \Omega_{2}$ we want to conclude that we have $\delta J_{1} \equiv 0$ (and hence $\delta J_{2} \equiv 0$ as well). Indeed, by Lemma A4, any pole of the differential $\delta J_{1}$ has to be a ramification point of $\Sigma_{E}$ and is of the order 1. By Lemma A3, at least $2 N-4$ zeroes of $\Omega_{2}$ are elements of $\mathcal{C}_{E} \backslash E$. Now we have to distinguish between two cases. If $\Omega_{2}\left(E_{n}\right) \neq 0$ for any $1 \leq n \leq 2 N(n \neq N, N+1)$, then the differential $\Omega_{2}$ has $2 N-2$ zeroes which are all contained in $\Sigma^{*} \backslash\left(\left\{\lambda_{1}, \ldots, \lambda_{2 N}\right\} \cup\left\{\infty^{+}, \infty^{-}\right\}\right)$. By Lemma A3, the zeroes of $\Omega_{2}$ cannot be zeroes of $\Omega_{1}$ and therefore (A18) implies that they must be zeroes of $\delta J_{2}$. Moreover, by Lemma A4, the differential $\delta J_{1}$ vanishes at $\infty^{ \pm}$of order 2, whereas $\Omega_{2}$ has a pole of order 2. Thus $\delta J_{1} \cdot \Omega_{2}$ is holomorphic at $\infty^{ \pm}$. By (A18) above, $\delta J_{2} \cdot \Omega_{1}$ is then holomorphic at $\infty^{ \pm}$. Since $\Omega_{1}$ has a pole of order 1 at the points $\infty^{ \pm}$it follows that $\delta J_{2}$ vanishes at $\infty^{ \pm}$. Alltogether, the differential $\delta J_{2}$ has at least $2 N$ zeroes on $\Sigma_{E}$. On the other hand, by Lemma A4, $\delta J_{2}$ has at most $2 N-2$ poles (which are all simple). As $\Sigma^{*}$ is a compact Riemann surface, the meromorphic function $\delta J_{2}$ vanishes identically, and thus by (A18), $\delta J_{1}$ as well.

It remains to consider the case where there exists $1 \leq n \leq 2 N, n \neq N, N+1$, such that $\Omega_{2}\left(\lambda_{n}\right)=0$. By Lemma A4, $\delta J_{1}$ is then either holomorphic near $\lambda_{n}$ or has a pole of order 1 . Therefore $\delta J_{1} \cdot \Omega_{2}$ is holomorphic near $\lambda_{n}$. By (A18), $\delta J_{2} \cdot \Omega_{1}$ then is holomorphic at $\lambda_{n}$ as well. By Lemma A3, $\Omega_{1}\left(\lambda_{n}\right) \neq 0$, hence $\delta J_{2}$ is holomorphic near the point $\lambda_{n}$. Again by Lemma A4, it follows that $\delta J_{2}$ has at most $2 N-3$ poles in $\Sigma^{*}$. On the other hand, by Lemma A3, the differential $\delta J_{2}$ has at least $2 N-4$ zeroes in $\mathcal{C} \backslash\left\{\lambda_{1}, \ldots, \lambda_{2 N}\right\}$. We have already observed that $\delta J_{2}$ vanishes at $\infty^{+}$and $\infty^{-}$. Therefore $\delta J_{2}$ has at least $2 N-2$ zeroes and at most $2 N-3$ poles in $\Sigma^{*}$. Since $\Sigma^{*}$ is a compact Riemann surface, it follows that the meromorphic function $\delta J_{2}$ vanishes identically, and so does $\delta J_{1}$.

\section{References}

1. Fermi, E.; Pasta, J.; Ulam, S. Studies of non linear problems. In Collected Papers of Enrico Fermi, 2nd ed.; Theory, Methods and Applications; University of Chicago Press: Chicago, IL, USA, 1965; Volume 2, pp. $978-988$.

2. Tomei, C. The Toda lattice, old and new. J. Geom. Mech. 2013, 5, 511-530. [CrossRef]

3. Kostant, B. The solution to a generalized Toda lattice and representation theory. Adv. Math. 1979, 34, 195-338. [CrossRef]

4. Nakamura, J.; Sekido, H. An application of the discrete-time Toda lattice to the progressive algorithm by Lanczos and related problems. J. Phys. A 2018, 51, 174001. [CrossRef]

5. Mokross, F.; Buttner, H. Thermal conductivity in the diatomic Toda lattice. J. Phys. C 1983, 16, 45-81. [CrossRef]

6. Andersson, T.; Damgaard, P.H.; Splittorff, K. The replica method and Toda lattice equations for QCD3. Nucl. Phys. B 2004, 707, 509-528. [CrossRef]

7. Gaeta, G. Results and Limitations of the Soliton Theory of DNA Transcription. J. Biol. Phys. 1999, 24, 81-96. [CrossRef] [PubMed]

8. Kodama, Y.; Shipman, B. Fifty years of the finite nonperiodic Toda lattice: A geometric and topological viewpoint. J. Phys. A 2018, 51, 353001. [CrossRef]

9. Takasaki, K. Toda hierarchies and their applications. J. Phys. A 2018, 51, 203001. [CrossRef]

10. Suris, Y. Discrete time Toda systems. J. Phys. A 2018, 51, 333001. [CrossRef]

11. Berman, G.P.; Izrailev, F.M. The Fermi-Pasta-Ulam problem: Fifty years of progress. Chaos 2005, 15, 015104. [CrossRef]

12. Krüger, H.; Teschl, G. Long-Time Asymptotics of the Toda Lattice for Decaying Initial Data Revisited. Rev. Math. Phys. 2009, 21, 61-109. [CrossRef]

13. Gardner, C.S.; Greene, J.M.; Kruskal, M.D.; Miura, R.M. A method for solving the Korteweg-de Vries equation. Phys. Rev. Lett. 1967, 19, 1095-1097. [CrossRef]

14. Flaschka, H. The Toda lattice. I. Existence of integrals. Phys. Rev. B 1974, 9, 142-149. [CrossRef] 
15. Hénon, M. Integrals of the Toda lattice. Phys. Rev. Sect. B 1974, 9, 1924-1925. [CrossRef]

16. Manakov, S.V. Complete integrability and stochastization of discrete dynamical systems. Sov. Phys. JETP 1975, 40, 269-274.

17. Pöschel, J. Integrability of Hamiltonian systems on Cantor sets. Commun. Pure Appl. Math. 1982, 35, 653-695. [CrossRef]

18. Henrici, A. Symmetries of the periodic Toda lattice, with an application to normal forms and perurbations of the lattice with Dirichlet boundary conditions. Discret. Contin. Dyn. Syst. A 2015, 35, 2949-2977. [CrossRef]

19. Henrici, A.; Kappeler, T. Birkhoff normal form for the periodic Toda lattice. In Integrable Systems and Random Matrices; American Mathematical Society: Providence, RI, USA, 2008; Volume 458, pp. 11-29.

20. Pöschel, J. Nekhoroshev estimates for quasi-convex Hamiltonian systems. Math. Z. 1993, 213, $187-216$. [CrossRef]

21. Henrici, A.; Kappeler, T. Nekhoroshev theorem for the periodic Toda lattice. Chaos 2009, 19, 033120. [CrossRef] [PubMed]

22. Nekhoroshev, N.N. An exponential estimate of the time of stability of nearly-integrable Hamiltonian systems I. Russ. Math. Surv. 1977, 32, 1-65. [CrossRef]

23. Nekhoroshev, N.N. An exponential estimate of the time of stability of nearly-integrable Hamiltonian systems II. Trudy Sem. Petrovsk. 1979, 5, 5-50.

24. Grébert, B.; Kappeler, T. Symmetries of the Nonlinear Schrödinger Equation. Bull. Soc. Math. France 2002, 130, 603-618. [CrossRef]

25. Henrici, A.; Kappeler, T. Resonant normal form for even periodic FPU chains. J. Eur. Math. Soc. 2009, 11, 1025-1056. [CrossRef]

26. Rink, B. Proof of Nishida's conjecture on anharmonic lattices. Commun. Math. Phys. 2006, 261, 613-627. [CrossRef]

27. Bilman, D.; Nenciu, I. On the evolution of scattering data under perturbations of the Toda lattice. Physica D 2016, 330, 1-16. [CrossRef]

28. Bogoyavlensky, O.I. On perturbations of the periodic Toda lattice. Commun. Math. Phys. 1976, 3, $201-209$. [CrossRef]

29. Kamvissis, S.; Teschl, G. Stability of the periodic Toda lattice under short range perturbations. J. Math. Phys. 2012, 53, 073706. [CrossRef]

30. Kappeler, T.; Pöschel, J. KdV \& KAM; Ergebnisse der Mathematik, 3. Folge; Springer: Berlin, Germany, 2003; Volume 45.

31. Henrici, A.; Kappeler, T. Global Birkhoff coordinates for the periodic Toda lattice. Nonlinearity 2008, 21, 2731-2758. [CrossRef]

32. Rink, B. Symmetric invariant manifolds in the Fermi-Pasta-Ulam lattice. Physica D 2003, 175, 31-42. [CrossRef]

33. Toda, M. Theory of Nonlinear Lattices, 2nd ed.; Springer Series in Solid-State Sciences; Springer: Berlin, Germany, 1989.

34. Henrici, A.; Kappeler, T. Global action-angle variables for the periodic Toda lattice. Int. Math. Res. Not. 2008, 10. [CrossRef]

35. van Moerbeke, P. The spectrum of Jacobi matrices. Invent. Math. 1976, 37, 45-81. [CrossRef]

36. Kappeler, T.; Topalov, P. Global Well-Posedness of $\mathrm{KdV}$ in $H^{-1}(\mathbb{T}, \mathbb{R})$. Duke Math. J. 2006, 135, 327-360. [CrossRef]

37. Teschl, G. Jacobi Operators and Completely Integrable Nonlinear Lattices; Mathematical Surveys and Monographs; American Mathematical Society: Providence, RI, USA, 2000.

38. Bikbaev, R.F.; Kuksin, S.B. On the parametrization of finite-gap solutions by frequency and wavenumber vectors and a theorem of I. Krichever. Lett. Math. Phys. 1993, 28, 115-122. [CrossRef]

(C) 2018 by the author. Licensee MDPI, Basel, Switzerland. This article is an open access article distributed under the terms and conditions of the Creative Commons Attribution (CC BY) license (http:/ / creativecommons.org/licenses/by/4.0/). 